\title{
The Global Landscape of Phase Retrieval II: Quotient Intensity Models
}

\author{
Jian-Feng Cai ${ }^{1}$, Meng Huang ${ }^{1}$, Dong $\mathrm{Li}^{2}{ }^{2 *}$ and Yang Wang ${ }^{1}$ \\ ${ }^{1}$ Department of Mathematics, The Hong Kong University of Science and \\ Technology, Clear Water Bay, Kowloon, Hong Kong \\ ${ }^{2}$ SUSTech International Center for Mathematics and Department of \\ Mathematics, Southern University of Science and Technology, Shenzhen, \\ Guangdong 518055, China
}

Received 3 April 2021; Accepted (in revised version) 10 November 2021

\begin{abstract}
A fundamental problem in phase retrieval is to reconstruct an unknown signal from a set of magnitude-only measurements. In this work we introduce three novel quotient intensity models (QIMs) based on a deep modification of the traditional intensity-based models. A remarkable feature of the new loss functions is that the corresponding geometric landscape is benign under the optimal sampling complexity. When the measurements $a_{i} \in \mathbb{R}^{n}$ are Gaussian random vectors and the number of measurements $m \geq C n$, the QIMs admit no spurious local minimizers with high probability, i.e., the target solution $x$ is the unique local minimizer (up to a global phase) and the loss function has a negative directional curvature around each saddle point. Such benign geometric landscape allows the gradient descent methods to find the global solution $x$ (up to a global phase) without spectral initialization.
\end{abstract}

AMS subject classifications: 94A12, 65K10, 49K45

Key words: Phase retrieval, landscape analysis, non-convex optimization.

\section{Introduction}

\footnotetext{
${ }^{*}$ Corresponding author.

Emails: jfcai@ust.hk (J. Cai), menghuang@ust.hk (M. Huang), lid@sustech.edu.cn (D. Li), yangwang@ust.hk (Y. Wang)
} 


\subsection{Background}

The intensity-based model for phase retrieval is

$$
y_{j}=\left|a_{j} \cdot u\right|^{2}, \quad j=1, \cdots, m
$$

where $a_{j} \in \mathbb{R}^{n}, j=1, \cdots, m$ are given vectors and $m$ is the number of measurements. The phase retrieval problem aims to recover the unknown signal $x \in \mathbb{R}^{n}$ based on the measurements $\left\{\left(a_{j}, y_{j}\right)\right\}_{j=1}^{m}$. A natural approach to solve this problem is to consider the minimization problem

$$
\min _{u \in \mathbb{R}^{n}} f(u)=\frac{1}{m} \sum_{j=1}^{m}\left(\left(a_{j} \cdot u\right)^{2}-\left(a_{j} \cdot x\right)^{2}\right)^{2} .
$$

However, as shown in [28], to guarantee the above loss function to have benign geometric landscape, the requirement of sampling complexity is $\mathcal{O}\left(n \log ^{3} n\right)$. This result is recently improved to $\mathcal{O}(n \log n)$ in [6]. On the other hand, due to the heavy tail of the quartic random variables in (1.1), such results seem to be optimal for this class of loss functions.

To remedy this issue, we propose in this work three novel quotient intensity models (QIMs) to recover $x$ under optimal sampling complexity. We rigorously prove that, for Gaussian random measurements, those empirical loss functions admit the benign geometric landscapes with high probability under the optimal sampling complexity $\mathcal{O}(n)$. Here, the phrase "benign" means: (1) the loss function has no spurious local minimizers; and (2) the loss function has a negative directional curvature around each saddle point. The three quotient intensity models are

QIM1:

$$
\min _{u \in \mathbb{R}^{n}} f(u)=\frac{1}{m} \sum_{k=1}^{m} \frac{\left(\left(a_{k} \cdot u\right)^{2}-\left(a_{k} \cdot x\right)^{2}\right)^{2}}{\left(a_{k} \cdot x\right)^{2}} .
$$

QIM2:

$$
\min _{u \in \mathbb{R}^{n}} f(u)=\frac{1}{m} \sum_{k=1}^{m} \frac{\left(\left(a_{k} \cdot u\right)^{2}-\left(a_{k} \cdot x\right)^{2}\right)^{2}}{\beta\|u\|_{2}^{2}+\left(a_{k} \cdot x\right)^{2}} .
$$

QIM3:

$$
\min _{u \in \mathbb{R}^{n}} f(u)=\frac{1}{m} \sum_{k=1}^{m} \frac{\left(\left(a_{k} \cdot u\right)^{2}-\left(a_{k} \cdot x\right)^{2}\right)^{2}}{\|u\|_{2}^{2}+\beta_{1}\left(a_{k} \cdot u\right)^{2}+\beta_{2}\left(a_{k} \cdot x\right)^{2}} .
$$


The phase retrieval problem arises in many fields of science and engineering such as X-ray crystallography [17,23], microscopy [22], astronomy [8], coherent diffractive imaging $[16,27]$ and optics [33] etc. In practical applications due to the physical limitations optical detectors can only record the magnitude of signals while losing the phase information. Many algorithms have been designed to solve the phase retrieval problem, which includes convex algorithms and non-convex ones. The convex algorithms usually rely on a "matrix-lifting" technique, which is computationally inefficient for large scale problems $[2,4,32]$. In contrast, many non-convex algorithms bypass the lifting step and operate directly on the lower-dimensional ambient space, making them much more computationally efficient. Early non-convex algorithms were mostly based on the technique of alternating projections, e.g., GerchbergSaxton [15] and Fineup [10]. The main drawback, however, is the lack of theoretical guarantee. Later Netrapalli et al. [24] proposed the AltMinPhase algorithm based on a technique known as spectral initialization. They proved that the algorithm linearly converges to the true solution with $\mathcal{O}\left(n \log ^{3} n\right)$ resampling Gaussian random measurements. This work led further to several other non-convex algorithms based on spectral initialization. A common thread is first choosing a good initial guess through spectral initialization, and then solving an optimization model through gradient descent, such as the Wirtinger Flow method [3], Truncated Wirtinger Flow algorithm [7], randomized Kaczmarz method [18,30,35], Gauss-Newton method [12], Truncated Amplitude Flow algorithm [34], Reshaped Wirtinger Flow (RWF) [36] and so on.

\subsection{Prior arts and connections}

As was already mentioned earlier, producing a good initial guess using spectral initialization seems to be a prerequisite for prototypical non-convex algorithms to succeed with good theoretical guarantees. A natural and fundamental question is:

Is it possible for non-convex algorithms to achieve successful recovery with a random initialization (i.e., without spectral initialization or any additional truncation)?

In the recent work [28], Ju Sun et al. carried out a deep study of the global geometric structure of phase retrieval problem. They proved that the loss function does not have any spurious local minima under $\mathcal{O}\left(n \log ^{3} n\right)$ Gaussian random measurements. More specifically, it was shown in [28] that all local minimizers coincide with the target signal $\boldsymbol{x}$ up to a global phase, and the loss function has a negative directional curvature around each saddle point. Thanks to this benign geometric landscape any algorithm which can avoid saddle points converges to the true solution with high probability. A trust-region method was employed in [28] to find the global minimizers with random initialization. To reduce the sampling complexity, 
it has been shown in [21] that a combination of the loss function with a judiciously chosen activation function also possesses the benign geometry structure under $\mathcal{O}(n)$ Gaussian random measurements. Recently, a smoothed amplitude flow estimator has been proposed in [5] and the authors show that the loss function has benign geometry structure under the optimal sampling complexity. Numerical tests show that the estimator in [5] yields very stable and fast convergence with random initialization and performs as good as or even better than the existing gradient descent methods with spectral initialization.

The emerging concept of a benign geometric landscape has also recently been explored in many other applications of signal processing and machine learning, e.g., matrix sensing [1,25], tensor decomposition [13], dictionary learning [29] and matrix completion [14]. For general optimization problems there exist a plethora of loss functions with well-behaved geometric landscapes such that all local optima are also global optima and each saddle point has a negative direction curvature in its vincinity. Correspondingly several techniques have been developed to guarantee that the standard gradient based optimization algorithms can escape such saddle points efficiently, see e.g., $[9,19,20]$.

\subsection{Our contributions}

This paper aims to show the intensity-based model (1.1) with some deep modification has a benign geometry structure under the optimal sampling complexity. More specifically, we first introduce three novel quotient intensity models and then we prove rigorously that each loss function of them has no spurious local minimizers. Furthermore, the loss function of quotient intensity model has a negative directional curvature around each saddle point. Such properties allow first order method like gradient descent to locate a global minimum with random initial guess.

Our first result shows that the loss function of (1.2) has the benign geometric landscape, as stated below.

Theorem 1.1 (Informal). Consider the quotient intensity model (1.2). Assume $\left\{a_{i}\right\}_{i=1}^{m}$ are i.i.d. standard Gaussian random vectors and $x \neq 0$. There exist positive absolute constants $c, C$, such that if $m \geq C n$, then with probability at least $1-e^{-c m}$ the loss function $f=f(u)$ has no spurious local minimizers. The only local minimizers are $\pm x$. All other critical points are strict saddles.

The second result is the global analysis for the estimator (1.3).

Theorem 1.2 (Informal). Consider the quotient intensity model (1.3). Let $0<$ $\beta<\infty$. Assume $\left\{a_{i}\right\}_{i=1}^{m}$ are i.i.d. standard Gaussian random vectors and $x \neq 0$. There exist positive constants $c, C$ depending only on $\beta$, such that if $m \geq C n$, then 
with probability at least $1-e^{-c m}$ the loss function $f=f(u)$ has no spurious local minimizers. The only local minimizer is $\pm x$ and all other critical points are strict saddles.

Remark 1.1. There appears some subtle differences between estimators (1.2) and (1.3). Although the former looks more singular, one can prove full strong convexity in the neighborhood of the global minimizers. In the latter case, however, we only have certain restricted convexity.

The third result is the global landscape for the estimator (1.4).

Theorem 1.3 (Informal). Consider the quotient intensity model (1.4). Let $0<$ $\beta_{1}, \beta_{2}<\infty$. Assume $\left\{a_{i}\right\}_{i=1}^{m}$ are i.i.d. standard Gaussian random vectors and $x \neq 0$. There exist positive constants $c, C$ depending only on $\beta$, such that if $m \geq C n$, then with probability at least $1-e^{-c m}$ the loss function $f=f(u)$ has no spurious local minimizers. The only local minimizer is $\pm x$ and all other critical points are strict saddles.

Remark 1.2. For this case, thanks to the strong damping, we have full strong convexity in the neighborhood of the global minimizers.

\subsection{Notations}

Throughout this proof we fix $\beta>0$ as a constant and do not study the precise dependence of other parameters on $\beta$. We write $u \in \mathbb{S}^{n-1}$ if $u \in \mathbb{R}^{n}$ and $\|u\|_{2}=$ $\sqrt{\sum_{j} u_{j}^{2}}=1$. We use $\chi$ to denote the usual characteristic function. For example $\chi_{A}(x)=1$ if $x \in A$ and $\chi_{A}(x)=0$ if $x \notin A$. We denote by $\delta_{1}, \epsilon, \eta, \eta_{1}$ various constants whose value will be taken sufficiently small. The needed smallness will be clear from the context. For any quantity $X$, we shall write $X=\mathcal{O}(Y)$ if $|X| \leq C Y$ for some constant $C>0$. We write $X \lesssim Y$ if $X \leq C Y$ for some constant $C>0$. We shall write $X \ll Y$ if $X \leq c Y$ where the constant $c>0$ will be sufficiently small. In our proof it is important for us to specify the precise dependence of the sampling size $m$ in terms of the dimension $n$. For this purpose we shall write $m \gtrsim n$ if $m \geq C n$ where the constant $C$ is allowed to depend on $\beta$ and the small constants $\epsilon, \epsilon_{i}$ etc used in the argument. One can extract more explicit dependence of $C$ on the small constants and $\beta$ but for simplicity we suppress this dependence here. We shall say an event $A$ happens with high probability if $\mathbb{P}(A) \geq 1-C e^{-c m}$, where $c>0, C>0$ are constants. The constants $c$ and $C$ are allowed to depend on $\beta$ and the small constants $\epsilon, \delta$ mentioned before. 


\subsection{Organization}

In Sections 2-4 we carry out an in-depth analysis of the corresponding geometric landscape of QIM1, QIM2 and QIM3 under optimal sampling complexity $\mathcal{O}(n)$. In Section 5, we report some numerical experiments to demonstrate the efficiency of our proposed estimators. In Appendix, we collect the technique lemmas which are used in the proof.

\section{Quotient intensity model I}

In this section, we consider the first quotient intensity model and prove that it has benign geometric landscape, as demonstrated below.

$$
f(u)=\frac{1}{m} \sum_{k=1}^{m} \frac{\left(\left(a_{k} \cdot u\right)^{2}-\left(a_{k} \cdot x\right)^{2}\right)^{2}}{\left(a_{k} \cdot x\right)^{2}} .
$$

Theorem 2.1. Assume $\left\{a_{k}\right\}_{k=1}^{m}$ are i.i.d. standard Gaussian random vectors and $x \neq 0$. There exist positive absolute constants $c, C$, such that if $m \geq C n$, then with probability at least $1-e^{-c m}$ the loss function $f=f(u)$ defined by $(2.1)$ has no spurious local minimizers. The only local minimizer is $\pm x$, and the loss function is strongly convex in a neighborhood of $\pm x$. The point $u=0$ is a local maximum point with strictly negative-definite Hessian. All other critical points are strict saddles, i.e., each saddle point has a neighborhood where the function has negative directional curvature.

Without loss of generality we shall assume $x=e_{1}$ throughout the rest of the proof. Note that the set $\bigcup_{k=1}^{m}\left\{a_{k} \cdot e_{1}=0\right\}$ has measure zero. Thus for typical realization we have $a_{k} \cdot e_{1} \neq 0$ for all $k$. This means that the loss function $f(u)$ defined by $(2.1)$ is smooth almost surely. We denote the Hessian of the function $f(u)$ along the $\xi$-direction $\left(\xi \in \mathbb{S}^{n-1}\right)$ as

$$
H_{\xi \xi}(u)=\sum_{i, j=1}^{n} \xi_{i} \xi_{j}\left(\partial_{i j} f\right)(u)=\frac{4}{m} \sum_{k=1}^{m}\left(3 \frac{\left(a_{k} \cdot \xi\right)^{2}\left(a_{k} \cdot u\right)^{2}}{\left(a_{k} \cdot e_{1}\right)^{2}}-\left(a_{k} \cdot \xi\right)^{2}\right) .
$$

\subsection{Strong convexity near the global minimizers $u= \pm e_{1}$}

Theorem 2.2 (Strong convexity near $u= \pm e_{1}$ ). There exists an absolute constant $0<\epsilon_{0} \ll 1$ such that the following hold. For $m \gtrsim n$, it holds with high probability that

$$
H_{\xi \xi}(u) \geq 1, \quad \forall \xi \in \mathbb{S}^{n-1}, \quad \forall u \quad \text { with } \quad\left\|u \pm e_{1}\right\|_{2} \leq \epsilon_{0} .
$$


Proof. By Lemma A.1, we can take $\epsilon>0$ sufficiently small, $N$ sufficiently large such that

$$
\mathbb{E} \frac{\left(a_{k} \cdot \xi\right)^{2}\left(a_{k} \cdot e_{1}\right)^{2}}{\epsilon+\left(a_{k} \cdot e_{1}\right)^{2}} \phi\left(\frac{a_{k} \cdot \xi}{N}\right) \geq 0.99, \quad \forall \xi \in \mathbb{S}^{n-1}, \quad \forall 1 \leq k \leq m
$$

In the above $\phi \in C_{c}^{\infty}(\mathbb{R})$ satisfies $0 \leq \phi(x) \leq 1$ for all $x, \phi(x)=1$ for $|x| \leq 1$ and $\phi(x)=0$ for $|x| \geq 2$. Clearly if $\left\|u \pm e_{1}\right\|_{2} \leq \epsilon_{0}$ and $\epsilon_{0}$ is sufficiently small (depending on $\epsilon$ and $N)$, then

$$
\mathbb{E} \frac{\left(a_{k} \cdot \xi\right)^{2}\left(a_{k} \cdot u\right)^{2}}{\epsilon+\left(a_{k} \cdot e_{1}\right)^{2}} \phi\left(\frac{a_{k} \cdot \xi}{N}\right) \geq 0.98, \quad \forall \xi \in \mathbb{S}^{n-1}, \quad \forall 1 \leq k \leq m
$$

The above term inside the expectation is clearly OK for union bounds. Thus for $\left\|u \pm e_{1}\right\| \leq \epsilon_{0}$ and $m \gtrsim n$, it holds with high probability that

$$
\frac{1}{4} H_{\xi \xi}(u) \geq \frac{1}{m} \sum_{k=1}^{m}\left(\frac{\left(a_{k} \cdot \xi\right)^{2}\left(a_{k} \cdot u\right)^{2}}{\epsilon+\left(a_{k} \cdot e_{1}\right)^{2}} \phi\left(\frac{a_{k} \cdot \xi}{N}\right)-\left(a_{k} \cdot \xi\right)^{2}\right) \geq 3 \cdot 0.97-1.01, \quad \forall \xi \in \mathbb{S}^{n-1}
$$

Thus the desired inequality follows.

\subsection{The regimes $\|u\|_{2} \ll 1$ and $\|u\|_{2} \gg 1$ are fine}

We first investigate the point $u=0$. It is trivial to verify that $\nabla f(0)=0$ since $a_{k} \cdot e_{1} \neq 0$ for all $k$ almost surely.

Lemma 2.1 ( $u=0$ has strictly negative-definite Hessian). We have $u=0$ is a local maximum point with strictly negative-definite Hessian. More precisely, for $m \gtrsim n$, it holds with high probability that

$$
\sum_{k, l=1}^{n} \xi_{k} \xi_{l}\left(\partial_{k l} f\right)(0) \leq-1, \quad \forall \xi \in \mathbb{S}^{n-1}
$$

Proof. By (2.2), it is obvious that

$$
H_{\xi \xi}(0)=-4 \frac{1}{m} \sum_{k=1}^{m}\left(a_{k} \cdot \xi\right)^{2}
$$

The desired conclusion then easily follows from Bernstein's inequality. 
Write $u=\sqrt{R} \hat{u}$ where $\hat{u} \in S^{n-1}$ and $R>0$. Then

$$
f(u)=\frac{1}{m} \sum_{k=1}^{m} \frac{\left(R\left(a_{k} \cdot \hat{u}\right)^{2}-\left(a_{k} \cdot e_{1}\right)^{2}\right)^{2}}{\left(a_{k} \cdot e_{1}\right)^{2}} .
$$

A simple calculation leads to

$$
\begin{aligned}
& \partial_{R} f=2 R \frac{1}{m} \sum_{k=1}^{m} \frac{\left(a_{k} \cdot \hat{u}\right)^{4}}{\left(a_{k} \cdot e_{1}\right)^{2}}-2 \frac{1}{m} \sum_{k=1}^{m}\left(a_{k} \cdot \hat{u}\right)^{2} \\
& \partial_{R R} f=2 \frac{1}{m} \sum_{k=1}^{m} \frac{\left(a_{k} \cdot \hat{u}\right)^{4}}{\left(a_{k} \cdot e_{1}\right)^{2}} .
\end{aligned}
$$

Lemma 2.2 (The regime $\|u\|_{2} \geq 1+\epsilon_{0}$ is $\mathrm{OK}$ ). Let $0<\epsilon_{0} \ll 1$ be any given small constant. Then the following hold: For $m \gtrsim n$, with high probability it holds that

$$
\partial_{R} f>0, \quad \forall R \geq 1+\epsilon_{0}, \quad \forall \hat{u} \in \mathbb{S}^{n-1} .
$$

Proof. Denote $X_{k}=a_{k} \cdot e_{1}$ and $Z_{k}=a_{k} \cdot \hat{u}$. By (2.3a) and Cauchy-Schwartz, we have

$$
\begin{aligned}
\partial_{R} f & \geq \frac{2 R}{m} \frac{\left(\sum_{k=1}^{m}\left(a_{k} \cdot \hat{u}\right)^{2}\right)^{2}}{\sum_{k=1}^{m}\left(a_{k} \cdot e_{1}\right)^{2}}-\frac{2}{m} \sum_{k=1}^{m}\left(a_{k} \cdot \hat{u}\right)^{2} \\
& \geq 2 R \cdot\left(1-\delta_{1}\right)-2\left(1+\delta_{1}\right), \quad \forall \hat{u} \in \mathbb{S}^{n-1},
\end{aligned}
$$

where $0<\delta_{1} \ll 1$ is an absolute constant which we can take to be sufficiently small, and in the last inequality we have used Bernstein. The desired result then easily follows by taking $R \geq R_{1}=\frac{1+2 \delta_{1}}{1-\delta_{1}}$ and choosing $\delta_{1}$ such that $R_{1} \leq 1+\epsilon_{0}$.

From (2.3a), due to the highly irregular coefficients near $R$, it is difficult to control the upper bound of $\partial_{R} f$ in the regime $R \ll 1$. To resolve this difficulty, we shall examine the Hessian in this regime.

Lemma 2.3 (The regime $\|u\|_{2} \leq \frac{1}{3}$ is $\mathrm{OK}$ ). For $m \gtrsim n$, with high probability it holds that

$$
H_{e_{1} e_{1}}(u) \leq-\frac{1}{2}<0, \quad \forall 0<\|u\|_{2} \leq \frac{1}{3},
$$

where $H_{e_{1} e_{1}}$ is defined in (2.2). 
Proof. It follows from (2.2) together with Bernstein's inequality that for $m \gtrsim n$ with high probability, it holds

$$
\frac{1}{4} H_{e_{1} e_{1}}(u)=\frac{1}{m} \sum_{k=1}^{m}\left(3\left(a_{k} \cdot u\right)^{2}-\left(a_{k} \cdot e_{1}\right)^{2}\right) \leq\|u\|_{2}^{2} \cdot 3 \cdot \frac{10}{9}-\frac{8}{9} \leq-\frac{1}{2} .
$$

This completes the proof.

Theorem 2.3 (The regimes $\|u\|_{2} \leq \frac{1}{3}$ and $\|u\|_{2} \geq 1+\epsilon_{0}$ are OK). Let $0<\epsilon_{0} \ll 1$ be a given small constant. For $m \gtrsim n$, with high probability the following hold:

1. We have

$$
\partial_{R} f>0, \quad \forall R \geq 1+\epsilon_{0}, \quad \forall \hat{u} \in \mathbb{S}^{n-1} .
$$

2. The point $u=0$ is a local maximum point with strictly negative-definite Hessian,

$$
\sum_{k, l=1}^{n} \xi_{k} \xi_{l}\left(\partial_{k l} f\right)(0) \leq-1, \quad \forall \xi \in \mathbb{S}^{n-1}
$$

3. We have

$$
H_{e_{1} e_{1}}(u) \leq-1, \quad \forall\|u\|_{2} \leq \frac{1}{3}
$$

Proof. This follows from Lemmas 2.1, 2.2 and 2.3.

Theorem 2.4 (The regime $\|u\|_{2} \sim 1,|| \hat{u} \cdot e_{1}|-1| \geq \eta_{0}$ ). Let $0<\eta_{0} \ll 1$ be given. Then for $m \gtrsim n$, the following hold with high probability: Suppose $u=\sqrt{R} \hat{u}, 1 / 9 \leq R \leq 2$, and ||$\hat{u} \cdot e_{1}|-1| \geq \eta_{0}$. If $\left(\partial_{R} f\right)(u)=0$, then we must have

$$
H_{e_{1} e_{1}}(u)<0 .
$$

Proof. By (2.3a), we have if $\partial_{R} f(u)=0$, then

$$
R \frac{1}{m} \sum_{k=1}^{m} \frac{\left(a_{k} \cdot \hat{u}\right)^{4}}{\left(a_{k} \cdot e_{1}\right)^{2}}=\frac{1}{m} \sum_{k=1}^{m}\left(a_{k} \cdot \hat{u}\right)^{2} .
$$

By Lemma A.2, we have for $m \gtrsim n$, it holds with high probability that

$$
\frac{1}{m} \sum_{k=1}^{m} \frac{\left(a_{k} \cdot \hat{u}\right)^{4}}{\left(a_{k} \cdot e_{1}\right)^{2}} \geq 100, \quad \forall \hat{u} \in \mathbb{S}^{n-1} \text { with }|| \hat{u} \cdot e_{1}|-1| \geq \eta_{0} .
$$

Clearly then $R \leq \frac{1}{50}$ with high probability. Thus it follows easily that $H_{e_{1} e_{1}}(u)<0$ also with high probability. 
Theorem 2.5 (Localization of $R$ when ||$\hat{u} \cdot e_{1}|-1| \leq \eta_{0}, R \leq 1+\eta_{0}$ and $u$ is a critical point). Let $0<\eta_{0} \ll 1$ be given. For $m \gtrsim n$, the following hold with high probability: Assume $u=\sqrt{R} \hat{u}$ is a critical point with $\frac{1}{9} \leq R \leq 1+\eta_{0}$, and ||$\hat{u} \cdot e_{1}|-1| \leq \eta_{0}$. Then we must have

$$
|R-1| \leq c\left(\eta_{0}\right)
$$

where $c\left(\eta_{0}\right) \rightarrow 0$ as $\eta_{0} \rightarrow 0$.

Proof. Denote $\partial_{\xi} f=\xi \cdot \nabla f$ for $\xi \in \mathbb{S}^{n-1}$. It is not difficult to check that

$$
\frac{1}{4} \partial_{\xi} f=\frac{1}{m} \sum_{k=1}^{m} \frac{\left(a_{k} \cdot u\right)^{3}\left(a_{k} \cdot \xi\right)}{X_{k}^{2}}-\frac{1}{m} \sum_{k=1}^{m}\left(a_{k} \cdot u\right)\left(a_{k} \cdot \xi\right)=0,
$$

where $X_{k}=a_{k} \cdot e_{1}$. Setting $\xi=\hat{u}$ and $\xi=e_{1}$, respectively give us two equations:

$$
\begin{aligned}
& R \cdot\left(\frac{1}{m} \sum_{k=1}^{m} \frac{\left(a_{k} \cdot \hat{u}\right)^{4}}{X_{k}^{2}}\right)-\frac{1}{m} \sum_{k=1}^{m}\left(a_{k} \cdot \hat{u}\right)^{2}=0, \\
& R \cdot\left(\frac{1}{m} \sum_{k=1}^{m} \frac{\left(a_{k} \cdot \hat{u}\right)^{3}}{X_{k}}\right)-\frac{1}{m} \sum_{k=1}^{m}\left(a_{k} \cdot \hat{u}\right) X_{k}=0 .
\end{aligned}
$$

We then obtain

$$
\left(\frac{1}{m} \sum_{k=1}^{m}\left(a_{k} \cdot \hat{u}\right)^{2}\right) \cdot\left(\frac{1}{m} \sum_{k=1}^{m} \frac{\left(a_{k} \cdot \hat{u}\right)^{3}}{X_{k}}\right)=\left(\frac{1}{m} \sum_{k=1}^{m} \frac{\left(a_{k} \cdot \hat{u}\right)^{4}}{X_{k}^{2}}\right) \cdot\left(\frac{1}{m} \sum_{k=1}^{m}\left(a_{k} \cdot \hat{u}\right) X_{k}\right) .
$$

Without loss of generality we assume $\left\|\hat{u}-e_{1}\right\|_{2} \leq \eta \ll 1$. Then with high probability we have

$$
\begin{aligned}
& \frac{1}{m} \sum_{k=1}^{m}\left(a_{k} \cdot \hat{u}\right) X_{k}=1+\mathcal{O}(\eta), \\
& \frac{1}{m} \sum_{k=1}^{m}\left(a_{k} \cdot \hat{u}\right)^{2}=1+\mathcal{O}(\eta) .
\end{aligned}
$$

Observe that by Cauchy-Schwartz,

$$
\sum_{k=1}^{m} \frac{\left|a_{k} \cdot \hat{u}\right|^{3}}{\left|X_{k}\right|} \leq\left(\sum_{k=1}^{m} \frac{\left|a_{k} \cdot \hat{u}\right|^{4}}{X_{k}^{2}}\right)^{\frac{1}{2}} \cdot\left(\sum_{k=1}^{m}\left(a_{k} \cdot \hat{u}\right)^{2}\right)^{\frac{1}{2}}
$$


Plugging the above estimates into (2.5), we obtain

$$
\sqrt{\frac{1}{m} \sum_{k=1}^{m} \frac{\left(a_{k} \cdot \hat{u}\right)^{4}}{X_{k}^{2}}} \leq 1+\mathcal{O}(\eta) .
$$

Using (2.4a), we then get

$$
R \geq 1+\mathcal{O}(\eta)
$$

The desired result then easily follows.

We now complete the proof of the main theorem.

Proof of Theorem 2.1. We proceed in several steps.

1. By Theorem 2.2, the function $f(u)$ is strongly convex when $\left\|u \pm e_{1}\right\|_{2} \ll 1$.

2. By Theorem 2.3, $f$ has non-vanishing gradient when $R \geq 1+\epsilon_{0}$. Also $H_{e_{1} e_{1}}(u) \leq$ -1 when $\|u\|_{2} \leq \frac{1}{3}$. The point $u=0$ is a strict local maximum point with strictly negative-definite Hessian.

3. By Theorem 2.4, we have $H_{e_{1} e_{1}}(u)<0$ if $\|u\|_{2} \sim 1$ and $\| \hat{u} \cdot e_{1}|-1| \geq \epsilon_{0}$.

4. Theorem 2.5 shows that if $R \leq 1+\epsilon_{0},|| \hat{u} \cdot e_{1}|-1| \leq \epsilon_{0}$ and $u$ is a critical point, then we must have $|R-1| \leq c\left(\epsilon_{0}\right) \ll 1$. In yet other words we must have $\left\|u \pm e_{1}\right\|_{2} \ll 1$. This regime is then treated by Step 1 .

This completes the proof.

\section{Quotient intensity model II}

Consider for $\beta>0$,

$$
f(u)=\frac{1}{m} \sum_{k=1}^{m} \frac{\left(\left(a_{k} \cdot u\right)^{2}-\left(a_{k} \cdot x\right)^{2}\right)^{2}}{\beta\|u\|_{2}^{2}+\left(a_{k} \cdot x\right)^{2}} .
$$

Theorem 3.1. Let $0<\beta<\infty$. Assume $\left\{a_{k}\right\}_{k=1}^{m}$ are i.i.d. standard Gaussian random vectors and $x \neq 0$. There exist positive constants $c, C$ depending only on $\beta$, such that if $m \geq C n$, then with probability at least $1-e^{-c m}$ the loss function $f=f(u)$ defined by (3.1) has no spurious local minimizers. The only local minimizer is $\pm x$, and the loss function is restrictively convex in a neighborhood of $\pm x$. The point $u=0$ is a local maximum point with strictly negative-definite Hessian. All other critical points are strict saddles, i.e., each saddle point has a neighborhood where the function has negative directional curvature. 
Remark 3.1. See Theorem 3.4 for the precise statement concerning restrictive convexity.

Without loss of generality we shall assume $x=e_{1}$ throughout the rest of the proof.

\subsection{The regimes $\|u\|_{2} \ll 1$ and $\|u\|_{2} \gg 1$ are fine}

We first investigate the point $u=0$. It is trivial to verify that $\nabla f(0)=0$ since $a_{k} \cdot e_{1} \neq 0$ for all $k$ almost surely.

Lemma 3.1 ( $u=0$ has strictly negative-definite Hessian). We have $u=0$ is local maximum point with strictly negative-definite Hessian. More precisely, for $m \gtrsim n$, it holds with high probability that

$$
\sum_{k, l=1}^{n} \xi_{k} \xi_{l}\left(\partial_{k l} f\right)(0) \leq-d_{1}, \quad \forall \xi \in \mathbb{S}^{n-1},
$$

where $d_{1}>0$ is an absolute constant.

Proof. We begin by noting that since almost surely $a_{k} \cdot e_{1} \neq 0$ for all $k$, the function $f$ is smooth at $u=0$. It suffices for us to consider (write $u=\sqrt{t} \xi$ )

$$
G(t)=\frac{1}{m} \sum_{k=1}^{m} \frac{\left(t\left(a_{k} \cdot \xi\right)^{2}-\left(a_{k} \cdot e_{1}\right)^{2}\right)^{2}}{\beta t+\left(a_{k} \cdot e_{1}\right)^{2}} .
$$

Clearly

$$
G^{\prime}(0)=-\beta-2 \frac{1}{m} \sum_{k=1}^{m}\left(a_{k} \cdot \xi\right)^{2} .
$$

The desired conclusion then easily follows by using Bernstein's inequality.

Write $u=\sqrt{R} \hat{u}$ where $\hat{u} \in S^{n-1}$ and $R>0$. Then

$$
f(u)=\frac{1}{m} \sum_{k=1}^{m} \frac{\left(R\left(a_{k} \cdot \hat{u}\right)^{2}-\left(a_{k} \cdot e_{1}\right)^{2}\right)^{2}}{\beta R+\left(a_{k} \cdot e_{1}\right)^{2}} .
$$

Clearly

$$
\begin{aligned}
& \partial_{R} f=\frac{1}{m} \sum_{k=1}^{m} \frac{R^{2}\left(\beta\left(a_{k} \cdot \hat{u}\right)^{4}\right)+2 R\left(a_{k} \cdot \hat{u}\right)^{4}\left(a_{k} \cdot e_{1}\right)^{2}-\beta\left(a_{k} \cdot e_{1}\right)^{4}-2\left(a_{k} \cdot e_{1}\right)^{4}\left(a_{k} \cdot \hat{u}\right)^{2}}{\left(\beta R+\left(a_{k} \cdot e_{1}\right)^{2}\right)^{2}} \\
& \partial_{R R} f=2 \frac{1}{m} \sum_{k=1}^{m} \frac{\left(a_{k} \cdot e_{1}\right)^{4}\left(\beta+\left(a_{k} \cdot \hat{u}\right)^{2}\right)^{2}}{\left(\beta R+\left(a_{k} \cdot e_{1}\right)^{2}\right)^{3}}
\end{aligned}
$$


Lemma 3.2 (The regime $\|u\|_{2} \gg 1$ is $\mathrm{OK}$ ). There exist constants $R_{1}=R_{1}(\beta)>0$, $d_{1}=d_{1}(\beta)>0$ such that the following hold: For $m \gtrsim n$, with high probability it holds that

$$
\partial_{R} f \geq d_{1}, \quad \forall R \geq R_{1}, \quad \forall \hat{u} \in \mathbb{S}^{n-1} .
$$

Proof. We only sketch the proof. Denote $X_{k}=a_{k} \cdot e_{1}$ and $Z_{k}=a_{k} \cdot \hat{u}$. Using the inequalities (assume $R \gg 1$ and denote by $C_{1}>0$ a constant depending only on $\beta$ )

$$
\beta R+X_{k}^{2} \leq R\left(\beta+X_{k}^{2}\right), \quad\left(\beta R+X_{k}^{2}\right)^{2} \geq 4 \beta R X_{k}^{2},
$$

and

$$
\frac{X_{k}^{4}}{\left(\beta R+X_{k}^{2}\right)^{2}} \leq C_{1} \cdot\left(\frac{R}{R^{2}}+\chi_{\left|X_{k}\right| \geq R^{\frac{1}{4}}}\right)
$$

we have

$$
\partial_{R} f \geq \frac{1}{m} \sum_{k=1}^{m} \frac{\beta Z_{k}^{4}}{\left(\beta+X_{k}^{2}\right)^{2}} \phi\left(\frac{Z_{k}}{N}\right)-\frac{1}{m} \sum_{k=1}^{m} \frac{1}{4 R} X_{k}^{2}-\frac{2}{m} \sum_{k=1}^{m} C_{1} \cdot\left(R^{-1}+\chi_{\left|X_{k}\right| \geq R^{\frac{1}{4}}}\right) \cdot Z_{k}^{2},
$$

where we have chosen $\phi \in C_{c}^{\infty}$ such that $0 \leq \phi(x) \leq 1$ for all $x, \phi(x)=1$ for $|x| \leq 1$ and $\phi(x)=0$ for $|x| \geq 2$. Observe that for $a \sim \mathcal{N}\left(0, \mathrm{I}_{\mathrm{n}}\right), Z \sim \mathcal{N}(0,1)$,

$$
\mathbb{E}(a \cdot \hat{u})^{4} \chi_{|a \cdot \hat{u}| \geq N} \leq \mathbb{E} Z^{4} \chi_{|Z| \geq N} \rightarrow 0 \quad \text { as } \quad N \rightarrow \infty .
$$

It is also easy to show that

$$
\inf _{\hat{u} \in \mathbb{S}^{n-1}} \mathbb{E} \frac{(a \cdot \hat{u})^{4}}{\left(\beta+\left(a \cdot e_{1}\right)^{2}\right)^{2}} \gtrsim 1
$$

Thus we can take $N$ large such that

$$
\inf _{\hat{u} \in \mathbb{S}^{n-1}} \mathbb{E} \frac{(a \cdot \hat{u})^{4}}{\left(\beta+\left(a \cdot e_{1}\right)^{2}\right)^{2}} \phi\left(\frac{a \cdot \hat{u}}{N}\right) \gtrsim 1 .
$$

It is easy to show that by taking $R$ large, for $m \gtrsim n$, it holds with high probability that

$$
\frac{1}{m} \sum_{k=1}^{m} \chi_{\left|X_{k}\right| \geq R^{\frac{1}{4}}} Z_{k}^{2} \leq \epsilon
$$

Since all the other terms are OK for union bounds, the desired result then clearly follows by taking $R$ large. 
Lemma 3.3 (The regime $\|u\|_{2} \ll 1$ with $\frac{\left|u_{1}\right|}{\|u\|_{2}} \leq \frac{1}{10}$ is $\mathrm{OK}$ ). There exist a constant $R_{2}=R_{2}(\beta)>0$ such that the following hold: For $m \gtrsim n$, with high probability it holds that

$$
\partial_{u_{1} u_{1}} f \leq-2<0, \quad \forall 0<R \leq R_{2}, \quad \forall \hat{u} \in \mathbb{S}^{n-1} \text { with }\left|\hat{u} \cdot e_{1}\right| \leq \frac{1}{10} .
$$

Proof. We only sketch the proof. Denote $X_{k}=a_{k} \cdot e_{1}$ and $Z_{k}=a_{k} \cdot \hat{u}$. A short computation gives

$$
\begin{aligned}
\partial_{u_{1} u_{1}} f=\frac{4}{m} & \sum_{k=1}^{m} \frac{3 R X_{k}^{2} Z_{k}^{2}-X_{k}^{4}}{\beta R+X_{k}^{2}}+\frac{1}{m} \sum_{k=1}^{m}\left(R Z_{k}^{2}-X_{k}^{2}\right)^{2} \cdot \frac{6 \beta^{2} u_{1}^{2}-2 \beta^{2}\left|u^{\prime}\right|^{2}-2 \beta X_{k}^{2}}{\left(\beta R+X_{k}^{2}\right)^{3}} \\
& -16 \beta\left(\hat{u} \cdot e_{1}\right) R^{2} \frac{1}{m} \sum_{k=1}^{m} \frac{Z_{k}^{3} X_{k}}{\left(\beta R+X_{k}^{2}\right)^{2}}+16 \beta\left(\hat{u} \cdot e_{1}\right) R \frac{1}{m} \sum_{k=1}^{m} \frac{X_{k}^{3} Z_{k}}{\left(\beta R+X_{k}^{2}\right)^{2}},
\end{aligned}
$$

where $u_{1}=u \cdot e_{1}$ and $u^{\prime}=u-u_{1} e_{1}$. Now observe that

$$
\begin{aligned}
& \frac{1}{m} \sum_{k=1}^{m} \frac{Z_{k}^{2}}{\beta R+X_{k}^{2}} X_{k}^{2} \leq \frac{1}{m} \sum_{k=1}^{m} Z_{k}^{2} ; \\
& \frac{1}{m} \sum_{k=1}^{m} \frac{X_{k}^{4}}{\beta R+X_{k}^{2}}=\frac{1}{m} \sum_{k=1}^{m} \frac{\left(\beta R+X_{k}^{2}-\beta R\right)^{2}}{\beta R+X_{k}^{2}} \\
& \geq\left(\frac{1}{m} \sum_{k=1}^{m}\left(\beta R+X_{k}^{2}\right)\right)-2 \beta R \geq-\beta R+\frac{1}{m} \sum_{k=1}^{m} X_{k}^{2} ; \\
& \frac{1}{m} \sum_{k=1}^{m} \frac{R^{\frac{3}{2}}\left|Z_{k}\right|^{3} R^{\frac{1}{2}}\left|X_{k}\right|}{\left(\beta R+X_{k}^{2}\right)^{2}} \leq \epsilon_{1} \frac{1}{m} \sum_{k=1}^{m} \frac{R^{2} Z_{k}^{4}}{\left(\beta R+X_{k}^{2}\right)^{2}}+\frac{1}{\epsilon_{1}^{3}} \frac{1}{m} \sum_{k=1}^{m} \frac{R^{2} X_{k}^{4}}{\left(\beta R+X_{k}^{2}\right)^{2}} \\
& \leq \frac{R^{2}}{\epsilon_{1}^{3}}+\epsilon_{1} \frac{1}{m} \sum_{k=1}^{m} \frac{R^{2} Z_{k}^{4}}{\left(\beta R+X_{k}^{2}\right)^{2}} ; \\
& \frac{R}{m} \sum_{k=1}^{m} \frac{\left|X_{k}\right|^{3}\left|Z_{k}\right|}{\left(\beta R+X_{k}^{2}\right)^{2}} \leq \frac{R}{m} \sum_{k=1}^{m} \frac{\left|X_{k}\right|^{3}\left|Z_{k}\right|}{\left(3(\beta R)^{\frac{1}{3}}\left(\frac{1}{4} X_{k}^{4}\right)^{\frac{1}{3}}\right)^{2}} \\
& \lesssim R^{\frac{1}{3}} \beta^{-\frac{2}{3}} \frac{1}{m} \sum_{k=1}^{m}\left|X_{k}\right|^{\frac{1}{3}}\left|Z_{k}\right| \lesssim R^{\frac{1}{3}} \beta^{-\frac{2}{3}} \frac{1}{m} \sum_{k=1}^{m}\left(X_{k}^{2}+Z_{k}^{2}+1\right),
\end{aligned}
$$

where in the above the constant $\epsilon_{1}>0$ will be taken sufficiently small. The needed smallness will become clear momentarily. Since $\left|u_{1}\right| /\|u\|_{2} \leq \frac{1}{10}$, it is clear that for some absolute constant $C_{1}>0$,

$$
\frac{6 \beta^{2} u_{1}^{2}-2 \beta^{2}\left\|u^{\prime}\right\|_{2}^{2}-2 \beta X_{k}^{2}}{\left(\beta R+X_{k}^{2}\right)^{3}} \leq-\beta C_{1} \cdot \frac{1}{\left(\beta R+X_{k}^{2}\right)^{2}}
$$


Now

$$
-\frac{1}{m} \sum_{k=1}^{m} \frac{\left(R Z_{k}^{2}-X_{k}^{2}\right)^{2}}{\left(\beta R+X_{k}^{2}\right)^{2}} \leq-\frac{1}{m} \sum_{k=1}^{m} \frac{R^{2} Z_{k}^{4}}{\left(\beta R+X_{k}^{2}\right)^{2}}+\frac{2 R}{m} \sum_{k=1}^{m} \frac{Z_{k}^{2}}{\beta R+X_{k}^{2}} .
$$

Now take $\epsilon_{1}=\frac{C_{1}}{1000}$. By Lemma B.1, we can take $R$ sufficiently small such that with high probability

$$
\frac{2 R}{m} \sum_{k=1}^{m} \frac{Z_{k}^{2}}{\beta R+X_{k}^{2}}<\frac{1}{100}
$$

All the other terms can be treated by taking $R$ sufficiently small, and the desired result follows easily.

Lemma 3.4 (The regime $\|u\|_{2} \ll 1$ with $\frac{\left|u_{1}\right|}{\|u\|_{2}}>\frac{1}{10}$ is OK). There exist a constant $R_{3}=R_{3}(\beta)>0$ such that the following hold: For $m \gtrsim n$, with high probability it holds that the loss function $f=f(u)$ has no critical points in the regime

$$
\left\{u=\sqrt{R} \hat{u}: 0<R \leq R_{3}, \hat{u} \in \mathbb{S}^{n-1} \text { and }\left|\hat{u} \cdot e_{1}\right|>\frac{1}{10}\right\}
$$

Proof. We assume that for $0<R \ll 1$ there exists some critical point. The idea is to examine the necessary conditions for a potential critical point and then derive a lower bound on $R$. Denote $X_{k}=a_{k} \cdot e_{1}$ and $Z_{k}=a_{k} \cdot \hat{u}$. By (3.2), we have $\partial_{R} f=0$ which gives

$$
\underbrace{R \frac{1}{m} \sum_{k=1}^{m} \frac{R \beta Z_{k}^{4}+2 Z_{k}^{4} X_{k}^{2}}{\left(\beta R+X_{k}^{2}\right)^{2}}}_{=: A_{1}}=\underbrace{\frac{1}{m} \sum_{k=1}^{m} \frac{\beta X_{k}^{4}+2 X_{k}^{4} Z_{k}^{2}}{\left(\beta R+X_{k}^{2}\right)^{2}}}_{=: B_{1}} .
$$

On the other hand, by using $\partial_{u_{1}} f(u)=0$, we obtain

$$
\begin{aligned}
& \beta u_{1} R^{2} \frac{1}{m} \sum_{k=1}^{m} \frac{Z_{k}^{4}}{\left(\beta R+X_{k}^{2}\right)^{2}}-2 R^{\frac{3}{2}} \frac{1}{m} \sum_{k=1}^{m} \frac{Z_{k}^{3} X_{k}}{\beta R+X_{k}^{2}} \\
= & \beta u_{1} \frac{1}{m} \sum_{k=1}^{m} \frac{2 R Z_{k}^{2} X_{k}^{2}-X_{k}^{4}}{\left(\beta R+X_{k}^{2}\right)^{2}}-2 R^{\frac{1}{2}} \frac{1}{m} \sum_{k=1}^{m} \frac{Z_{k} X_{k}^{3}}{\beta R+X_{k}^{2}} .
\end{aligned}
$$


Thus

$$
\begin{gathered}
-\underbrace{-R \widehat{u}_{1} \frac{1}{m} \sum_{k=1}^{m} \frac{\beta R Z_{k}^{4}}{\left(\beta R+X_{k}^{2}\right)^{2}}}_{=: A_{2}}+R \cdot \underbrace{2 \frac{1}{m} \sum_{k=1}^{m} \frac{Z_{k}^{3} X_{k}}{\beta R+X_{k}^{2}}}_{=: A_{3}} \\
=\underbrace{\beta \widehat{u}_{1} \frac{1}{m} \sum_{k=1}^{m} \frac{-2 R Z_{k}^{2} X_{k}^{2}+X_{k}^{4}}{\left(\beta R+X_{k}^{2}\right)^{2}}+2 \frac{1}{m} \sum_{k=1}^{m} \frac{Z_{k} X_{k}^{3}}{\beta R+X_{k}^{2}}}_{=: B_{2}} .
\end{gathered}
$$

Without loss of generality we assume $\widehat{u}_{1}>\frac{1}{10}$. Observe that for $0<R \leq 1$, we have

$$
\frac{1}{m} \sum_{k=1}^{m} \frac{X_{k}^{4}}{\left(\beta+X_{k}^{2}\right)^{2}} \lesssim B_{1} \lesssim 1+\frac{1}{m} \sum_{k=1}^{m} Z_{k}^{2}
$$

Thus with high probability $B_{1} \sim 1$.

Now by Lemma B.1, for $0<R \ll 1$, we have

$$
\frac{1}{m} \sum_{k=1}^{m} \frac{R Z_{k}^{2} X_{k}^{2}}{\left(\beta R+X_{k}^{2}\right)^{2}} \leq \frac{1}{m} \sum_{k=1}^{m} \frac{R Z_{k}^{2}}{\beta R+X_{k}^{2}} \ll 1 .
$$

Also for $0<R \leq 1$, we have

$$
\frac{1}{m} \sum_{k=1}^{m} \frac{X_{k}^{4}}{\left(\beta+X_{k}^{2}\right)^{2}} \leq \frac{1}{m} \sum_{k=1}^{m} \frac{X_{k}^{4}}{\left(\beta R+X_{k}^{2}\right)^{2}} \leq 1 .
$$

By Lemma B.2, for $0<R \ll 1$, we have

$$
c_{1} \leq \frac{1}{m} \sum_{k=1}^{m} \frac{Z_{k} X_{k}^{3}}{\beta R+X_{k}^{2}} \leq c_{2},
$$

where $c_{1}, c_{2}>0$ are constants depending only on $\beta$. Thus with high probability we have for $0<R \ll 1, B_{2} \sim 1$.

Now since

$$
R A_{1}=B_{1}, \quad-R A_{2}+R A_{3}=B_{2}
$$

we obtain

$$
A_{1}+B_{3} A_{2}=B_{3} A_{3}
$$


where $B_{3}=B_{1} / B_{2}$. Observe that $A_{1}>0, A_{2}>0$, and

$$
\begin{aligned}
& A_{1} \sim \frac{1}{m} \sum_{k=1}^{m} \frac{Z_{k}^{4}}{\beta R+X_{k}^{2}} ; \\
& A_{3} \leq\left(\frac{1}{m} \sum_{k=1}^{m} \frac{Z_{k}^{4}}{\beta R+X_{k}^{2}}\right)^{\frac{3}{4}}\left(\frac{1}{m} \sum_{k=1}^{m} \frac{X_{k}^{4}}{\beta R+X_{k}^{2}}\right)^{\frac{1}{4}} .
\end{aligned}
$$

It follows easily that with high probability we have

$$
A_{1} \sim 1
$$

But then it follows from the equation $R A_{1}=B_{1}$ that we must have $R \sim 1$. Thus the desired result follows.

Theorem 3.2 (The regimes $\|u\|_{2} \ll 1$ and $\|u\|_{2} \gg 1$ are OK). For $m \gtrsim n$, with high probability the following hold:

1. We have

$$
\partial_{R} f \geq d_{1}, \quad \forall R \geq R_{1}, \quad \forall \hat{u} \in \mathbb{S}^{n-1},
$$

where $d_{1}, R_{1}$ are constants depending only on $\beta$.

2. We have

$$
\partial_{u_{1} u_{1}} f \leq-2<0, \quad \forall 0<R \leq R_{2}, \quad \forall \hat{u} \in \mathbb{S}^{n-1} \quad \text { with }\left|\hat{u} \cdot e_{1}\right| \leq \frac{1}{10},
$$

where $R_{2}>0$ is a constant depending only on $\beta$.

3. The loss function $f=f(u)$ has no critical points in the regime

$$
\left\{u=\sqrt{R} \hat{u}: 0<R \leq R_{3}, \hat{u} \in \mathbb{S}^{n-1} \text { and }\left|\hat{u} \cdot e_{1}\right|>\frac{1}{10}\right\},
$$

where $R_{3}>0$ is a constant depending only on $\beta$.

4. The point $u=0$ is a local maximum point with strictly negative-definite Hessian,

$$
\sum_{k, l=1}^{n} \xi_{k} \xi_{l}\left(\partial_{k l} f\right)(0) \leq-d_{2}<0, \quad \forall \xi \in \mathbb{S}^{n-1},
$$

where $d_{2}>0$ is an absolute constant.

Proof. This follows from Lemmas 3.1, 3.2, 3.3 and 3.4. 


\subsection{The regime $\|u\|_{2} \sim 1$}

Lemma 3.5 (The regime $\|u\|_{2} \sim 1$ with $\epsilon_{0} \leq\left|\hat{u} \cdot e_{1}\right| \leq 1-\epsilon_{0}$ is OK). Let $0<\epsilon_{0} \ll 1$ be given. Assume $0<c_{1}<c_{2}<\infty$ are two given constants. Then for $m \gtrsim n$, the following hold with high probability: The loss function $f=f(u)$ has no critical points in the regime:

$$
\left\{u=\sqrt{R} \hat{u}: c_{1}<R<c_{2}, \epsilon_{0} \leq\left|\hat{u} \cdot e_{1}\right| \leq 1-\epsilon_{0}\right\}
$$

More precisely, introduce the parametrization $\hat{u}=e_{1} \cos \theta+e^{\perp} \sin \theta$, where $\theta \in[0, \pi]$ and $e^{\perp} \in \mathbb{S}^{n-1}$ satisfies $e^{\perp} \cdot e_{1}=0$. Then in the aforementioned regime, we have

$$
\left|\partial_{\theta} f\right| \geq \alpha_{1}>0
$$

where $\alpha_{1}$ depends only on $\left(\beta, \epsilon_{0}, c_{1}, c_{2}\right)$.

Proof. See appendix.

Lemma 3.6 (The regime $\|u\|_{2} \sim 1$ with $\left|\hat{u} \cdot e_{1}\right| \leq \epsilon_{1}$ is OK). Let $0<\epsilon_{1} \ll 1$ be a sufficiently small constant. Assume $0<c_{1}<c_{2}<\infty$ are two given constants. Then for $m \gtrsim n$, the following hold with high probability: Consider the regime

$$
\left\{u=\sqrt{R} \hat{u}: c_{1}<R<c_{2},\left|\hat{u} \cdot e_{1}\right| \leq \epsilon_{1}\right\} .
$$

Introduce the parametrization $\hat{u}=e_{1} \cos \theta+e^{\perp} \sin \theta$, where $\theta \in[0, \pi]$ and $e^{\perp} \in \mathbb{S}^{n-1}$ satisfies $e^{\perp} \cdot e_{1}=0$. Then in the aforementioned regime, we have

$$
\partial_{\theta \theta} f \leq-\alpha_{2}<0
$$

where $\alpha_{2}>0$ depends only on $\left(\beta, \epsilon_{1}, c_{1}, c_{2}\right)$.

Proof. See appendix.

Theorem 3.3 (The regime $\|u\|_{2} \sim 1,\left\|\hat{u} \cdot e_{1}|-1| \leq \epsilon_{0},\left|\|u\|_{2}-1\right| \geq c\left(\epsilon_{0}\right)\right.$ is OK). Let $0<R_{1}<1<R_{2}<\infty$ be given constants. Let $0<\epsilon_{0} \ll 1$ be a given sufficiently small constant and consider the regime ||$\hat{u} \cdot e_{1}|-1| \leq \epsilon_{0}$ with $R_{1} \leq\|u\|_{2}^{2} \leq R_{2}$. There exists a constant $c_{0}=c_{0}\left(\epsilon_{0}, R_{1}, R_{2}, \beta\right)>0$ which tends to zero as $\epsilon_{0} \rightarrow 0$ such that the following hold: For $m \gtrsim n$, with high probability it holds that (below $u=\sqrt{R} \hat{u}$ )

$$
\begin{array}{lllll}
\partial_{R} f<0, & \forall R_{2} \leq R \leq 1-c_{0}, & \forall \hat{u} \in \mathbb{S}^{n-1} & \text { with } & || \hat{u} \cdot e_{1}|-1| \leq \epsilon_{0} \\
\partial_{R} f>0, & \forall 1+c_{0} \leq R \leq R_{1}, & \forall \hat{u} \in \mathbb{S}^{n-1} & \text { with } & || \hat{u} \cdot e_{1}|-1| \leq \epsilon_{0}
\end{array}
$$


Proof. We first consider the regime $R \geq 1+c$. Let $\phi \in C_{c}^{\infty}(\mathbb{R})$ be an even function satisfying $0 \leq \phi(x) \leq 1$ for all $x, \phi(x)=1$ for $|x| \leq 1$ and $\phi(x)=0$ for $|x|>2$. By using (3.2), we have

$$
\geq \frac{1}{m} \sum_{k=1}^{m} \frac{R^{2} \beta\left(a_{k} \cdot \hat{u}\right)^{4} \phi\left(\frac{a_{k} \cdot \hat{u}}{K}\right)+2 R\left(a_{k} \cdot \hat{u}\right)^{4} \phi\left(\frac{a_{k} \cdot \hat{u}}{K}\right)\left(a_{k} \cdot e_{1}\right)^{2}-\beta\left(a_{k} \cdot e_{1}\right)^{4}-2\left(a_{k} \cdot e_{1}\right)^{4}\left(a_{k} \cdot \hat{u}\right)^{2}}{\left(\beta R+\left(a_{k} \cdot e_{1}\right)^{2}\right)^{2}} .
$$

By taking $K$ sufficiently large, we can easily obtain

$$
\mathbb{E}\left(1-\phi\left(\frac{a \cdot e_{1}}{K}\right)\right)\left(1+\left(a \cdot e_{1}\right)^{2}\right) \ll 1,
$$

where $a \sim \mathcal{N}\left(0, \mathrm{I}_{\mathrm{n}}\right)$. For fixed $K$, it is not difficult to check that the lower bound (3.4) are OK for union bounds and they can be made close to the expectation with high probability, uniformly in $R \sim 1$ and $\hat{u} \in \mathbb{S}^{n-1}$. The perturbation argument (i.e., estimating the error terms coming from replacing $a_{k} \cdot \hat{u}$ by $a_{k} \cdot e_{1}$ and so on) becomes rather easy after taking the expectation. It is then not difficult to show that

$$
\partial_{R} f>0
$$

for $R \geq 1+c\left(\epsilon_{0}\right)$.

Next we turn to the regime $R_{2} \leq R \leq 1-c\left(\epsilon_{0}\right)$. Without loss of generality we may assume $\left|1-\hat{u} \cdot e_{1}\right| \leq \epsilon_{0}$. The idea is to exploit the decomposition used in the proof of Lemma 3.4. Namely using $\partial_{R} f=0$ and $\partial_{u_{1}} f=0$, we have

$$
\begin{aligned}
& \underbrace{R \frac{1}{m} \sum_{k=1}^{m} \frac{R \beta Z_{k}^{4}+2 Z_{k}^{4} X_{k}^{2}}{\left(\beta R+X_{k}^{2}\right)^{2}}}_{=: A_{1}} \\
& =\underbrace{\frac{1}{m} \sum_{k=1}^{m} \frac{\beta X_{k}^{4}+2 X_{k}^{4} Z_{k}^{2}}{\left(\beta R+X_{k}^{2}\right)^{2}}}_{=: B_{1}} ;-R \underbrace{\widehat{u}_{1} \frac{1}{m} \sum_{k=1}^{m} \frac{\beta R Z_{k}^{4}}{\left(\beta R+X_{k}^{2}\right)^{2}}}_{=: A_{2}}+R \cdot \underbrace{2 \frac{1}{m} \sum_{k=1}^{m} \frac{Z_{k}^{3} X_{k}}{\beta R+X_{k}^{2}}}_{=: A_{3}} \\
& =\underbrace{\beta \widehat{u}_{1} \frac{1}{m} \sum_{k=1}^{m} \frac{-2 R Z_{k}^{2} X_{k}^{2}+X_{k}^{4}}{\left(\beta R+X_{k}^{2}\right)^{2}}+2 \frac{1}{m} \sum_{k=1}^{m} \frac{Z_{k} X_{k}^{3}}{\beta R+X_{k}^{2}}}_{=: B_{2}} \text {. }
\end{aligned}
$$

It is not difficult to check that with high probability, we have $B_{1} \sim 1, B_{2} \sim 1$, and

$$
\left|\frac{B_{2}}{B_{1}}-1\right| \leq \eta\left(\epsilon_{0}\right) \ll 1, \quad \forall R_{2} \leq R \leq 1, \quad \forall \hat{u} \in \mathbb{S}^{n-1} \text { with }\left|\hat{u} \cdot e_{1}-1\right| \leq \epsilon_{0}
$$


where $\eta\left(\epsilon_{0}\right) \rightarrow 0$ as $\epsilon_{0} \rightarrow 0$. We then obtain

$$
A_{1}=\left(1+\mathcal{O}\left(\eta\left(\epsilon_{0}\right)\right)\right)\left(-A_{2}+A_{3}\right)
$$

From this it is easy (similar to an argument used in the proof of Lemma 3.4) to derive that

$$
A_{1}+A_{2}+\left|A_{3}\right| \lesssim 1
$$

Now note that the pre-factor of $A_{2}$ is $\hat{u}_{1}=1+\mathcal{O}\left(\epsilon_{0}\right)$. By using the relation

$$
A_{1}+A_{2}-A_{3}=\mathcal{O}\left(\eta\left(\epsilon_{0}\right)\right)
$$

we obtain

$$
\frac{1}{m} \sum_{k=1}^{m} \frac{Z_{k}^{4}}{\beta R+X_{k}^{2}}-\frac{1}{m} \sum_{k=1}^{m} \frac{Z_{k}^{3} X_{k}}{\beta R+X_{k}^{2}}=\mathcal{O}\left(\eta\left(\epsilon_{0}\right)\right)
$$

By using localization, i.e., decomposing

$$
Z_{k}^{3} X_{k}=Z_{k}^{3} \phi\left(\frac{Z_{k}}{M}\right) X_{k}+Z_{k}^{3}\left(1-\phi\left(\frac{Z_{k}}{M}\right)\right) X_{k},
$$

Hölder and taking $M$ sufficiently large, one can then derive that (with high probability)

$$
\begin{aligned}
& \left|\frac{1}{m} \sum_{k=1}^{m} \frac{Z_{k}^{4}-X_{k}^{4}}{\beta R+X_{k}^{2}}\right|+\left|\frac{1}{m} \sum_{k=1}^{m} \frac{Z_{k}^{3} X_{k}-X_{k}^{4}}{\beta R+X_{k}^{2}}\right| \\
= & \mathcal{O}\left(\eta_{1}\left(\epsilon_{0}\right)\right), \quad \forall R_{2} \leq R \leq 1, \quad \forall \hat{u} \in \mathbb{S}^{n-1} \text { with }\left|\hat{u} \cdot e_{1}-1\right| \leq \epsilon_{0},
\end{aligned}
$$

where $\eta_{1}\left(\epsilon_{0}\right) \rightarrow 0$ as $\epsilon_{0} \rightarrow 0$. It then follows easily that (with high probability)

$$
\left|\frac{1}{m} \sum_{k=1}^{m} \frac{\left(Z_{k}-X_{k}\right)^{4}}{\beta R+X_{k}^{2}}\right|=\mathcal{O}\left(\eta_{2}\left(\epsilon_{0}\right)\right), \quad \forall R_{2} \leq R \leq 1, \quad \forall \hat{u} \in \mathbb{S}^{n-1} \text { with }\left|\hat{u} \cdot e_{1}-1\right| \leq \epsilon_{0}
$$

where $\eta_{2}\left(\epsilon_{0}\right) \rightarrow 0$ as $\epsilon_{0} \rightarrow 0$.

Now observe that for $A_{1}$, we have

$$
\begin{aligned}
\left|Z_{k}^{4}-X_{k}^{4}\right| & \leq\left|Z_{k}-X_{k}\right|\left(\mathcal{O}\left(\left|Z_{k}\right|^{3}\right)+\mathcal{O}\left(\left|X_{k}\right|^{3}\right)\right) \\
& \leq C_{\epsilon}\left|Z_{k}-X_{k}\right|^{4}+\epsilon \cdot\left(\mathcal{O}\left(\left|Z_{k}\right|^{4}\right)+\mathcal{O}\left(X_{k}^{4}\right)\right)
\end{aligned}
$$


where $C_{\epsilon}>0$ depends only on $\epsilon$. Clearly by taking $\epsilon>0$ sufficiently small and using the derived quantitative estimates preceding this paragraph, we can guarantee that (with high probability)

$$
\left|A_{1}-B_{1}\right| \ll 1, \quad \forall R_{2} \leq R \leq 1, \quad \forall \hat{u} \in \mathbb{S}^{n-1} \text { with }\left|\hat{u} \cdot e_{1}-1\right| \leq \epsilon_{0} .
$$

It follows that we must have $|R-1| \ll 1$ for a potential critical point. By using (3.2) we have $\partial_{R} f(R=0)<0$. By using (3.3) we have $\partial_{R R} f>0$. Since we have shown $\partial_{R} f>0$ for $R>1+c\left(\epsilon_{0}\right)$, it then follows that $\partial_{R} f=0$ occurs at a unique point $|R-1| \ll 1$ and $\partial_{R} f<0$ for $R<1-c\left(\epsilon_{0}\right)$ provided $c\left(\epsilon_{0}\right)$ is suitably re-defined.

We now show restrictive convexity of the loss function $f(u)$ near the global minimizer $u= \pm e_{1}$.

Theorem 3.4 (Restrictive convexity near the global minimizer). There exists $0<$ $\epsilon_{0} \ll 1$ sufficiently small such that if $m \gtrsim n$, then the following hold with high probability:

1. If $\left\|u-e_{1}\right\|_{2} \leq \epsilon_{0}$ and $u \neq e_{1}$, then for $\xi=\frac{u-e_{1}}{\left\|u-e_{1}\right\|_{2}} \in \mathbb{S}^{n-1}$, we have

$$
\sum_{i, j=1}^{n} \xi_{i} \xi_{j}\left(\partial_{i j} f\right)(u) \geq \gamma>0,
$$

where $\gamma$ is a constant depending only on $\beta$.

2. If $\left\|u+e_{1}\right\|_{2} \leq \epsilon_{0}$, then then for $\xi=\frac{u+e_{1}}{\left\|u+e_{1}\right\|_{2}} \in \mathbb{S}^{n-1}$, we have

$$
\sum_{i, j=1}^{n} \xi_{i} \xi_{j}\left(\partial_{i j} f\right)(u) \geq \gamma>0,
$$

where $\gamma$ is a constant depending only on $\beta$.

3. Alternatively we can use the parametrization $u= \pm e_{1}+t \xi$, where $\xi \in \mathbb{S}^{n-1}$, and $|t| \leq \epsilon_{0}$. Then with this special parametrization, we have

$$
\sum_{i, j=1}^{n} \xi_{i} \xi_{j}\left(\partial_{i j} f\right)(u) \geq \gamma>0 .
$$

Note that this includes the global minimizers $u= \pm e_{1}$.

In yet other words, $f(u)$ is restrictively convex in a sufficiently small neighborhood of $\pm e_{1}$. 
Proof. See appendix.

Proof of Theorem 3.1. We proceed in several steps as follows.

1. For the regime $\|u\|_{2} \ll 1$ and $\|u\|_{2} \gg 1$, we use Theorem 3.2. The point $u=0$ is a local maximum point with strictly negative-definite Hessian. All other possible critical points must have negative curvature direction.

2. For the regime $\|u\|_{2} \sim 1,|| \hat{u} \cdot e_{1}|-1| \geq \epsilon_{0}$, we use Lemma 3.5 and 3.6. The loss function either has a nonzero gradient, or it is a strict saddle with a negative curvature direction.

3. For the regime $\|u\|_{2} \sim 1,\left|\hat{u} \cdot e_{1}\right|-1\left|\leq \epsilon_{0},\right|\|u\|_{2}-1 \mid \geq c\left(\epsilon_{0}\right)$, we apply Theorem 3.3. The loss function has nonzero gradient in this regime.

4. Finally for the regime close to the global minimizers $\pm e_{1}$, we use Theorem 3.4 to show restrictive convexity. This ensures that $\pm e_{1}$ are the only minimizers.

This completes the proof.

\section{Quotient intensity model III}

Consider for $\beta_{1}>0, \beta_{2}>0$,

$$
f(u)=\frac{1}{m} \sum_{k=1}^{m} \frac{\left(\left(a_{k} \cdot u\right)^{2}-\left(a_{k} \cdot x\right)^{2}\right)^{2}}{\|u\|_{2}^{2}+\beta_{1}\left(a_{k} \cdot u\right)^{2}+\beta_{2}\left(a_{k} \cdot x\right)^{2}} .
$$

Theorem 4.1. Let $0<\beta_{1}, \beta_{2}<\infty$. Assume $\left\{a_{k}\right\}_{k=1}^{m}$ are i.i.d. standard Gaussian random vectors and $x \neq 0$. There exist positive constants $c, C$ depending only on $\left(\beta_{1}, \beta_{2}\right)$, such that if $m \geq C n$, then with probability at least $1-e^{-c m}$ the loss function $f=f(u)$ defined by (4.1) has no spurious local minimizers. The only local minimizer is $\pm x$, and the loss function is strongly convex in a neighborhood of $\pm x$. The point $u=0$ is a local maximum point with strictly negative-definite Hessian. All other critical points are strict saddles, i.e., each saddle point has a neighborhood where the function has negative directional curvature.

Without loss of generality we shall assume $x=e_{1}$ throughout the rest of the proof. Thus we consider

$$
f(u)=\frac{1}{m} \sum_{k=1}^{m} \frac{\left(\left(a_{k} \cdot u\right)^{2}-\left(a_{k} \cdot e_{1}\right)^{2}\right)^{2}}{\|u\|_{2}^{2}+\beta_{1}\left(a_{k} \cdot u\right)^{2}+\beta_{2}\left(a_{k} \cdot e_{1}\right)^{2}} .
$$




\subsection{The regimes $\|u\|_{2} \ll 1$ and $\|u\|_{2} \gg 1$ are fine}

We first investigate the point $u=0$. It is trivial to verify that $\nabla f(0)=0$ since $a_{k} \cdot e_{1} \neq 0$ for all $k$ almost surely.

Lemma 4.1 ( $u=0$ has strictly negative-definite Hessian). We have $u=0$ is a local maximum point with strictly negative-definite Hessian. More precisely, it holds (almost surely) that

$$
\sum_{k, l=1}^{n} \xi_{k} \xi_{l}\left(\partial_{k l} f\right)(0) \leq-d_{1}, \quad \forall \xi \in \mathbb{S}^{n-1}
$$

where $d_{1}>0$ is a constant depending only on $\beta_{2}$.

Proof. We begin by noting that since almost surely $a_{k} \cdot e_{1} \neq 0$ for all $k$, the function $f$ is smooth at $u=0$. It suffices for us to consider (write $u=\sqrt{t} \xi$ )

$$
G(t)=\frac{1}{m} \sum_{k=1}^{m} \frac{\left(t\left(a_{k} \cdot \xi\right)^{2}-\left(a_{k} \cdot e_{1}\right)^{2}\right)^{2}}{t+t \beta_{1}\left(a_{k} \cdot \xi\right)^{2}+\beta_{2}\left(a_{k} \cdot e_{1}\right)^{2}} .
$$

By a simple computation, we have

$$
G^{\prime}(0)=-\frac{1}{\beta_{2}^{2}}-\frac{\beta_{1}+2 \beta_{2}}{\beta_{2}^{2}} \cdot \frac{1}{m} \sum_{k=1}^{m}\left(a_{k} \cdot \xi\right)^{2} .
$$

The desired conclusion then easily follows.

Write $u=\sqrt{R} \hat{u}$ where $\hat{u} \in S^{n-1}$ and $R>0$. Denote $X_{k}=a_{k} \cdot e_{1}$. Then

$$
f(u)=\frac{1}{m} \sum_{k=1}^{m} \frac{\left(R\left(a_{k} \cdot \hat{u}\right)^{2}-X_{k}^{2}\right)^{2}}{R+\beta_{1} R\left(a_{k} \cdot \hat{u}\right)^{2}+\beta_{2} X_{k}^{2}} .
$$

Clearly

$$
\begin{aligned}
& \partial_{R} f=\frac{1}{m} \sum_{k=1}^{m} \frac{R^{2}\left(\left(a_{k} \cdot \hat{u}\right)^{4}+\beta_{1}\left(a_{k} \cdot \hat{u}\right)^{6}\right)+2 R \beta_{2}\left(a_{k} \cdot \hat{u}\right)^{4} X_{k}^{2}-X_{k}^{4}-\left(\beta_{1}+2 \beta_{2}\right)\left(a_{k} \cdot \hat{u}\right)^{2} X_{k}^{4}}{\left(R+R \beta_{1}\left(a_{k} \cdot \hat{u}\right)^{2}+\beta_{2} X_{k}^{2}\right)^{2}} ; \\
& \partial_{R R} f=2 \frac{1}{m} \sum_{k=1}^{m} \frac{\left(1+\left(a_{k} \cdot \hat{u}\right)^{2}\left(\beta_{1}+\beta_{2}\right)\right) X_{k}^{4}}{\left(R+R \beta_{1}\left(a_{k} \cdot \hat{u}\right)^{2}+\beta_{2} X_{k}^{2}\right)^{3}} .
\end{aligned}
$$


Lemma 4.2 (The regimes $\|u\|_{2} \gg 1$ or $\|u\|_{2} \ll 1$ are $\mathrm{OK}$ ). There exist constants $R_{i}=R_{i}\left(\beta_{1}, \beta_{2}\right)>0, d_{i}=d_{i}\left(\beta_{1}, \beta_{2}\right)>0, i=1,2$ such that the following hold: For $m \gtrsim n$, with high probability it holds that

$$
\begin{array}{lll}
\partial_{R} f \geq d_{1}, & \forall R \geq R_{1}, & \forall \hat{u} \in \mathbb{S}^{n-1} ; \\
\partial_{R} f \leq-d_{2}<0, & \forall 0<R \leq R_{2}, & \forall \hat{u} \in \mathbb{S}^{n-1} .
\end{array}
$$

Proof. Denote $Z_{k}=a_{k} \cdot \hat{u}$. We first consider the regime $R \gg 1$. Observe that

$$
\frac{1}{m} \sum_{k=1}^{m} \frac{R^{2} Z_{k}^{4}}{\left(R+R \beta_{1} Z_{k}^{2}+\beta_{2} X_{k}^{2}\right)^{2}} \gtrsim \frac{1}{m} \sum_{k=1}^{m} \frac{Z_{k}^{4}}{\left(1+Z_{k}^{2}+X_{k}^{2}\right)^{2}} \gtrsim 1, \quad \forall \hat{u} \in \mathbb{S}^{n-1},
$$

where the last inequality holds for $m \gtrsim n$ with high probability. On the other hand we note that

$$
\begin{aligned}
\frac{1}{m} \sum_{k=1}^{m} \frac{X_{k}^{4}}{\left(R+R \beta_{1} Z_{k}^{2}+\beta_{2} X_{k}^{2}\right)^{2}} & \lesssim \frac{1}{m} \sum_{k=1}^{m} \frac{X_{k}^{4}}{\left(R+X_{k}^{2}\right)^{2}}\left(\chi_{\left|X_{k}\right| \leq R^{\frac{1}{4}}}+\chi_{\left|X_{k}\right|>R^{\frac{1}{4}}}\right) \\
& \lesssim R^{-1}+\frac{1}{m} \sum_{k=1}^{m} \chi_{\left|X_{k}\right|>R^{\frac{1}{4}}} \ll 1, \quad \forall \hat{u} \in \mathbb{S}^{n-1},
\end{aligned}
$$

where again the last inequality holds for $R$ sufficiently large, and for $m \gtrsim n$ with high probability. Similarly we have for $R$ sufficiently large,

$$
\begin{aligned}
& \frac{1}{m} \sum_{k=1}^{m} \frac{\left(\beta_{1}+2 \beta_{2}\right) Z_{k}^{2} X_{k}^{4}}{\left(R+R \beta_{1} Z_{k}^{2}+\beta_{2} X_{k}^{2}\right)^{2}} \\
\lesssim & R^{-1} \frac{1}{m} \sum_{k=1}^{m} Z_{k}^{2}+\frac{1}{m} \sum_{k=1}^{m} Z_{k}^{2} \chi_{\left|X_{k}\right|>R^{\frac{1}{4}}} \ll 1, \quad \forall \hat{u} \in \mathbb{S}^{n-1} .
\end{aligned}
$$

Thus it follows easily that $\partial_{R} f \gtrsim 1$ for $R \gg 1$.

Now we turn to the regime $0<R \ll 1$. First we note that the main negative term is OK. This is due to the fact that for $0<R \leq 1$, we have (for $m \gtrsim n$ and with high probability)

$$
\frac{1}{m} \sum_{k=1}^{m} \frac{Z_{k}^{2} X_{k}^{4}}{\left(R+R Z_{k}^{2}+X_{k}^{2}\right)^{2}} \geq \frac{1}{m} \sum_{k=1}^{m} \frac{Z_{k}^{2} X_{k}^{4}}{\left(1+Z_{k}^{2}+X_{k}^{2}\right)^{2}} \gtrsim 1, \quad \forall \hat{u} \in \mathbb{S}^{n-1} .
$$


On the other hand, we have (for $m \gtrsim n$ and with high probability)

$$
\begin{aligned}
& \frac{1}{m} \sum_{k=1}^{m} \frac{R^{2}\left(Z_{k}^{4}+Z_{k}^{6}\right)}{\left(R+R Z_{k}^{2}+X_{k}^{2}\right)^{2}} \\
\leq & \frac{1}{m} \sum_{k=1}^{m} \frac{R Z_{k}^{4}}{R+R Z_{k}^{2}+X_{k}^{2}} \cdot\left(\chi_{\left|X_{k}\right| \geq R^{\frac{1}{4}}\left|Z_{k}\right|}+\chi_{\left.\left|X_{k}\right|<R^{\frac{1}{4}\left|Z_{k}\right|}\right)}\right) \\
\leq & R^{\frac{1}{2}} \frac{1}{m} \sum_{k=1}^{m} Z_{k}^{2}+\frac{1}{m} \sum_{k=1}^{m} Z_{k}^{2} \chi_{\left|X_{k}\right|<R^{\frac{1}{4}}\left|Z_{k}\right|} \\
\leq & R^{\frac{1}{2}} \frac{1}{m} \sum_{k=1}^{m} Z_{k}^{2}+\frac{1}{m} \sum_{k=1}^{m} Z_{k}^{2} \chi_{\left|Z_{k}\right| \geq K}+\frac{1}{m} \sum_{k=1}^{m} K^{2} \chi_{\left|X_{k}\right|<K R^{\frac{1}{4}}} \\
\ll & 1, \quad \forall \hat{u} \in \mathbb{S}^{n-1},
\end{aligned}
$$

if we first take $K$ sufficiently large followed by taking $R$ sufficiently small. The estimate of the other term $\frac{R Z_{k}^{4} X_{k}^{2}}{\left(R+R Z_{k}^{2}+X_{k}^{2}\right)^{2}}$ is similar and we omit further details.

Collecting the estimates, it is then clear that we can obtain the desired estimate for $\partial_{R} f$ when $0<R \ll 1$.

\subsection{The regime $\|u\|_{2} \sim 1$}

Lemma 4.3 (The regime $\|u\|_{2} \sim 1$ with $\epsilon_{0} \leq\left|\hat{u} \cdot e_{1}\right| \leq 1-\epsilon_{0}$ is OK). Let $0<\epsilon_{0} \ll 1$ be given. Assume $0<c_{1}<c_{2}<\infty$ are two given constants. Then for $m \gtrsim n$, the following hold with high probability: The loss function $f=f(u)$ has no critical points in the regime:

$$
\left\{u=\sqrt{R} \hat{u}: c_{1}<R<c_{2}, \epsilon_{0} \leq\left|\hat{u} \cdot e_{1}\right| \leq 1-\epsilon_{0}\right\} .
$$

More precisely, introduce the parametrization $\hat{u}=e_{1} \cos \theta+e^{\perp} \sin \theta$, where $\theta \in[0, \pi]$ and $e^{\perp} \in \mathbb{S}^{n-1}$ satisfies $e^{\perp} \cdot e_{1}=0$. Then in the aforementioned regime, we have

$$
\left|\partial_{\theta} f\right| \geq \alpha_{1}>0
$$

where $\alpha_{1}$ depends only on $\left(\beta, \epsilon_{0}, c_{1}, c_{2}\right)$.

Proof. We first recall

$$
f(u)=\frac{1}{m} \sum_{k=1}^{m} \frac{\left(R\left(a_{k} \cdot \hat{u}\right)^{2}-X_{k}^{2}\right)^{2}}{R+\beta_{1} R\left(a_{k} \cdot \hat{u}\right)^{2}+\beta_{2} X_{k}^{2}} .
$$


Clearly $a_{k} \cdot \hat{u}=X_{k} \cos \theta+\left(a_{k} \cdot e^{\perp}\right) \sin \theta$, and

$$
\begin{aligned}
& \partial_{\theta}\left(a_{k} \cdot \hat{u}\right)=X_{k}(-\sin \theta)+\left(a_{k} \cdot e^{\perp}\right) \cos \theta \\
& \partial_{\theta \theta}\left(a_{k} \cdot \hat{u}\right)=-\left(a_{k} \cdot \hat{u}\right) .
\end{aligned}
$$

In particular, if $\theta$ is away from the end-points $0, \pi$, then

$$
\partial_{\theta}\left(a_{k} \cdot \hat{u}\right)=\left(a_{k} \cdot \hat{u}\right) \cot \theta-X_{k} \csc \theta .
$$

We then obtain (below $Z_{k}=a_{k} \cdot \hat{u}$ )

$$
\begin{aligned}
\partial_{\theta} f=- & \csc \theta \frac{1}{m} \sum_{k=1}^{m} \frac{2 R Z_{k}\left(-X_{k}^{2}+R Z_{k}^{2}\right) \cdot\left(\left(\beta_{1}+2 \beta_{2}\right) X_{k}^{2}+R\left(2+\beta_{1} Z_{k}^{2}\right)\right) X_{k}}{\left(R+\beta_{1} R Z_{k}^{2}+\beta_{2} X_{k}^{2}\right)^{2}} \\
& +\cot \theta \frac{1}{m} \sum_{k=1}^{m} \frac{2 R Z_{k}\left(-X_{k}^{2}+R Z_{k}^{2}\right) \cdot\left(\left(\beta_{1}+2 \beta_{2}\right) X_{k}^{2}+R\left(2+\beta_{1} Z_{k}^{2}\right)\right) Z_{k}}{\left(R+\beta_{1} R Z_{k}^{2}+\beta_{2} X_{k}^{2}\right)^{2}} .
\end{aligned}
$$

Thanks to the strong damping, it is not difficult to check that for any $\epsilon>0$, if $m \gtrsim n$, then with high probability we have

$$
\left|\partial_{\theta} f-\mathbb{E} \partial_{\theta} f\right| \leq \epsilon, \quad \forall c_{1} \leq R \leq c_{2}, \quad \forall \hat{u} \in \mathbb{S}^{n-1}
$$

The desired result then follows from Lemma C.1.

Lemma 4.4 (The regime $\|u\|_{2} \sim 1$ with $\left|\hat{u} \cdot e_{1}\right| \leq \epsilon_{0}$ is OK). Let $0<\epsilon_{1} \ll 1$ be a sufficiently small constant. Assume $0<c_{1}<c_{2}<\infty$ are two given constants. Then for $m \gtrsim n$, the following hold with high probability: Consider the regime

$$
\left\{u=\sqrt{R} \hat{u}: c_{1}<R<c_{2},\left|\hat{u} \cdot e_{1}\right| \leq \epsilon_{1}\right\}
$$

Introduce the parametrization $\hat{u}=e_{1} \cos \theta+e^{\perp} \sin \theta$, where $\theta \in[0, \pi]$ and $e^{\perp} \in \mathbb{S}^{n-1}$ satisfies $e^{\perp} \cdot e_{1}=0$. Then in the aforementioned regime, we have

$$
\partial_{\theta \theta} f \leq-\alpha_{2}<0
$$

where $\alpha_{2}>0$ depends only on $\left(\beta, \epsilon_{1}, c_{1}, c_{2}\right)$.

Proof. This is similar to the argument in the proof of Lemma 4.3. By a tedious computation, we have

$$
\partial_{\theta \theta} f=\frac{1}{m} \sum_{k=1}^{m} \frac{2 R G_{k}}{\left(R+\beta_{2} x^{2}+\beta_{1} R Z_{k}^{2}\right)^{3}}
$$


where

$$
\begin{aligned}
G_{k}=- & 8 \beta_{1} R Z_{k}^{2}\left(-X_{k}^{2}+R Z_{k}^{2}\right)\left(R+\beta_{2} X_{k}^{2}+\beta_{1} R Z_{k}^{2}\right)\left(X_{k}-Z_{k} \cos \theta\right)^{2} \csc ^{2} \theta \\
& -2\left(R+\beta_{2} X_{k}^{2}+\beta_{1} R Z_{k}^{2}\right)^{2}\left(X_{k}^{4}-3 R X_{k}^{2} Z_{k}^{2}-R Z_{k}^{4}-2 X_{k} Z_{k}\left(X_{k}^{2}-3 R Z_{k}^{2}\right) \cos \theta\right. \\
& \left.+Z_{k}^{2}\left(X_{k}^{2}-2 R Z_{k}^{2}\right) \cos 2 \theta\right) \csc ^{2} \theta \\
& +\beta_{1}\left(X_{k}^{2}-R Z_{k}^{2}\right)^{2}\left(Z_{k}^{2}\left(R+\beta_{2} X_{k}^{2}+\beta_{1} R Z_{k}^{2}\right)+4 \beta_{1} R Z_{k}^{2}\left(X_{k}-Z_{k} \cos \theta\right)^{2} \csc ^{2} \theta\right. \\
& \left.-\left(R+\beta_{2} X_{k}^{2}+\beta_{1} R Z_{k}^{2}\right)\left(X_{k}-Z_{k} \cos \theta\right)^{2} \csc ^{2} \theta\right) .
\end{aligned}
$$

It is then tedious but not difficult to check that that for any $\epsilon>0$, if $m \gtrsim n$, then with high probability we have

$$
\left|\partial_{\theta \theta} f-\mathbb{E} \partial_{\theta \theta} f\right| \leq \epsilon, \quad \forall c_{1} \leq R \leq c_{2}, \quad \forall \hat{u} \in \mathbb{S}^{n-1} .
$$

The desired result then follows from Lemma C.1.

Theorem 4.2 (The regime $\|u\|_{2} \sim 1,\left\|\hat{u} \cdot e_{1}|-1| \leq \epsilon_{0},\left|\|u\|_{2}-1\right| \geq c\left(\epsilon_{0}\right)\right.$ is OK). Let $0<c_{1}<1<c_{2}<\infty$ be given constants. Let $0<\epsilon_{0} \ll 1$ be a given sufficiently small constant and consider the regime ||$\hat{u} \cdot e_{1}|-1| \leq \epsilon_{0}$ with $c_{1} \leq\|u\|_{2}^{2} \leq c_{2}$. There exists $a$ constant $c_{0}=c_{0}\left(\epsilon_{0}, c_{1}, c_{2}, \beta\right)>0$ which tends to zero as $\epsilon_{0} \rightarrow 0$ such that the following hold: For $m \gtrsim n$, with high probability it holds that (below $u=\sqrt{R} \hat{u}$ )

$$
\begin{aligned}
& \partial_{R} f<0, \quad \forall c_{2} \leq R \leq 1-c_{0}, \quad \forall \hat{u} \in \mathbb{S}^{n-1} \quad \text { with }|| \hat{u} \cdot e_{1}|-1| \leq \epsilon_{0} ; \\
& \partial_{R} f>0, \quad \forall 1+c_{0} \leq R \leq c_{1}, \quad \forall \hat{u} \in \mathbb{S}^{n-1} \quad \text { with }|| \hat{u} \cdot e_{1}|-1| \leq \epsilon_{0} .
\end{aligned}
$$

Proof. We rewrite

$$
f(u)=\frac{1}{m} \sum_{k=1}^{m} g\left(R,\left(a_{k} \cdot \hat{u}\right)^{2}, X_{k}^{2}\right),
$$

where

$$
g(R, a, b)=\frac{(R a-b)^{2}}{R+\beta_{1} R a+\beta_{2} b} .
$$

It is not difficult to check that for $R \sim 1$, we have

$$
\left|\left(\partial_{R} g\right)(R, a, b)-\left(\partial_{R} g\right)(R, b, b)\right| \leq\left\|\partial_{R a} g\right\|_{\infty}|b-a| \lesssim|b-a|, \quad \forall a, b \geq 0 .
$$

On the other hand, note that $\left(\partial_{R} g\right)(1, b, b)=0$, and for $R \sim 1$,

$$
\left(\partial_{R R} g\right)(R, b, b)=\frac{2 b^{2}\left(1+b\left(\beta_{1}+\beta_{2}\right)\right)^{2}}{\left(R+b\left(\beta_{2}+\beta_{1} R\right)\right)^{3}} \sim b .
$$


Thus for $R=1+\eta, \eta>0$ we have

$$
\begin{aligned}
\left(\partial_{R} g\right)(R, a, b) & \geq \partial_{R} g(R, b, b)-\gamma_{1}|b-a| \\
& \geq \gamma_{2} \cdot \eta \cdot b-\gamma_{1}|b-a|
\end{aligned}
$$

where $\gamma_{1}>0, \gamma_{2}>0$ are constants depending only on $\left(\beta_{1}, \beta_{2}, c_{1}, c_{2}\right)$. The desired result (for $\partial_{R} f>0$ when $R \rightarrow 1+$ ) then follows from this and simple application of Bernstein's inequalities. The estimate for the regime $R \rightarrow 1-$ is similar. We omit the details.

Theorem 4.3 (Strong convexity near the global minimizer). There exist $0<\epsilon_{0} \ll 1$ and a positive constant $\gamma$ such that if $m \gtrsim n$, then the following hold with high probability:

1. If $\left\|u-e_{1}\right\|_{2} \leq \epsilon_{0}$, then

$$
\sum_{i, j=1}^{n} \xi_{i} \xi_{j}\left(\partial_{i j} f\right)(u) \geq \gamma>0, \quad \forall \xi \in \mathbb{S}^{n-1} .
$$

2. If $\left\|u+e_{1}\right\|_{2} \leq \epsilon_{0}$, then

$$
\sum_{i, j=1}^{n} \xi_{i} \xi_{j}\left(\partial_{i j} f\right)(u) \geq \gamma>0, \quad \forall \xi \in \mathbb{S}^{n-1}
$$

In yet other words, $f(u)$ is strongly convex in a sufficiently small neighborhood of $\pm e_{1}$.

Proof. See appendix.

Finally we complete the proof of Theorem 4.1.

Proof of Theorem 4.1. We proceed in several steps. All the statements below hold under the assumption that $m \gtrsim n$ and with high probability.

1. For $u=0$, we use Lemma 4.1. In particular $u=0$ is a local maximum point with strictly negative Hessian.

2. For $\|u\|_{2} \ll 1$ or $\|u\|_{2} \gg 1$, we use Lemma 4.2. The loss functions has a nonzero gradient $\left(\partial_{R} f \neq 0\right)$ in this regime.

3. For $\|u\|_{2} \sim 1$ with $\epsilon_{0} \leq\left|\hat{u} \cdot e_{1}\right| \leq 1-\epsilon_{0}$, we use Lemma 4.3 to show that the loss function has a nonzero gradient $\left(\partial_{\theta} f \neq 0\right)$ in this regime. 
4. For $\|u\|_{2} \sim 1$ with $\left|\hat{u} \cdot e_{1}\right| \leq \epsilon_{0}$, by Lemma 4.4, the loss function has a negative curvature direction (i.e., $\partial_{\theta \theta} f<0$ ) in this regime.

5. For $\|u\|_{2} \sim 1,|| \hat{u} \cdot e_{1}|-1| \leq \epsilon_{0},\left|\|u\|_{2}-1\right| \geq c\left(\epsilon_{0}\right)$, Theorem 4.2 shows that the gradient of the loss function does not vanish (i.e., $\partial_{R} f \neq 0$ ).

6. For $\left\|u \pm e_{1}\right\| \ll 1$, Theorem 4.3 gives the strong convexity in the full neighborhood.

It is not difficult to check that the above 6 scenarios cover the whole of $\mathbb{R}^{n}$. We omit further details.

\section{$5 \quad$ Numerical experiments}

In this section, we demonstrate the numerical efficiency of our estimators by simple gradient descent and compare their performance with other competitive algorithms. Our Quotient intensity models are:

QIM1:

$$
\min _{u \in \mathbb{R}^{n}} f(u)=\frac{1}{m} \sum_{k=1}^{m} \frac{\left(\left(a_{k} \cdot u\right)^{2}-\left(a_{k} \cdot x\right)^{2}\right)^{2}}{\left(a_{k} \cdot x\right)^{2}} .
$$

QIM2:

$$
\min _{u \in \mathbb{R}^{n}} f(u)=\frac{1}{m} \sum_{k=1}^{m} \frac{\left(\left(a_{k} \cdot u\right)^{2}-\left(a_{k} \cdot x\right)^{2}\right)^{2}}{\beta\|u\|_{2}^{2}+\left(a_{k} \cdot x\right)^{2}} .
$$

QIM3:

$$
\min _{u \in \mathbb{R}^{n}} f(u)=\frac{1}{m} \sum_{k=1}^{m} \frac{\left(\left(a_{k} \cdot u\right)^{2}-\left(a_{k} \cdot x\right)^{2}\right)^{2}}{\|u\|_{2}^{2}+\beta_{1}\left(a_{k} \cdot u\right)^{2}+\beta_{2}\left(a_{k} \cdot x\right)^{2}} .
$$

We have shown theoretically that any gradient descent algorithm will not get trapped in a local minimum for the estimators above. Here we present numerical experiments to show that the estimators perform very well with randomized initial guess.

We test the performance of our QIM2 and QIM3 and compare with SAF [5], Trust Region [29], WF [3], TWF [7] and TAF [34]. Here, it is worth emphasizing that random initialization is used for SAF, Trust Region [29] and our QIM2, QIM3 algorithms while all other algorithms have adopted a spectral initialization. 


\section{$5.1 \quad$ Recovery of $1 \mathrm{D}$ signals}

In our numerical experiments, the target vector $x \in \mathbb{R}^{n}$ is chosen randomly from the standard Gaussian distribution and the measurement vectors $a_{i}, i=1, \cdots, m$ are generated randomly from standard Gaussian distribution or CDP model. For the real Gaussian case, the signal $x \sim \mathcal{N}\left(0, I_{n}\right)$ and measurement vectors $a_{i} \sim \mathcal{N}\left(0, I_{n}\right)$ for $i=1, \cdots, m$. For the complex Gaussian case, the signal $x \sim \mathcal{N}\left(0, I_{n}\right)+i \mathcal{N}\left(0, I_{n}\right)$ and measurement vectors $a_{i} \sim \mathcal{N}\left(0, I_{n} / 2\right)+i \mathcal{N}\left(0, I_{n} / 2\right)$. For the CDP model, we use masks of octanary patterns as in [3]. For simplicity, our parameters and step size are fixed for all experiments. Specifically, we adopt parameter $\beta=1$ and step size $\mu=0.4$ for QIM2 and choose the parameter $\beta_{1}=0.1, \beta_{2}=1$, step size $\mu=0.3$ for QIM3. For Trust Region, WF, TWF and TAF, we use the codes provided in the original papers with suggested parameters.

Example 5.1. In this example, we test the empirical success rate of QIM2, QIM3 versus the number of measurements. We conduct the experiments for the real Gaussian, complex Gaussian and CDP cases, respectively. We choose $n=128$ and the maximum number of iterations is $T=2500$. For real and complex Gaussian cases, we vary $m$ within the range $[n, 10 n]$. For CDP case, we set the ratio $m / n=L$ from 2 to 10 . For each $m$, we run 100 times trials to calculate the success rate. Here, we say a trial to have successfully reconstructed the target signal if the relative error satisfies $\operatorname{dist}\left(u_{T}-x\right) /\|x\| \leq 10^{-5}$. The results are plotted in Fig. 1. It can be seen that $6 n$ Gaussian phaseless measurement or 7 octanary patterns are enough for exactly recovery for QIM2 and QIM3.

Example 5.2. In this example, we compare the convergence rate of QIM2, QIM3 with those of SAF, WF, TWF, TAF for real Gaussian and complex Gaussian cases. We choose $n=128$ and $m=6 n$. The results are presented in Fig. 2. We can see that our algorithms perform well comparing with state-of-the-art algorithms with spectral initialization.

Example 5.3. In this example, we compare the time elapsed and the iteration needed for WF, TWF, TAF, SAF and our QIM2, QIM3 to achieve the relative error $10^{-5}$ and $10^{-10}$, respectively. We choose $n=1000$ with $m=8 n$. We adopt the same spectral initialization method for WF, TWF, TAF and the initial guess is obtained by power method with 50 iterations. We run 50 times trials to calculate the average time elapsed and iteration number for those algorithms. The results are shown in Table 1. The numerical results show that QIM3 takes around 27 and 50 iterations to escape the saddle points for the real and complex Gaussian cases, respectively. 


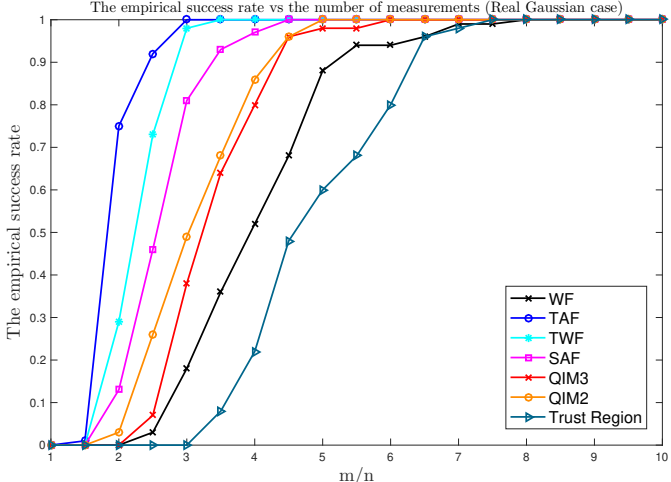

(a)

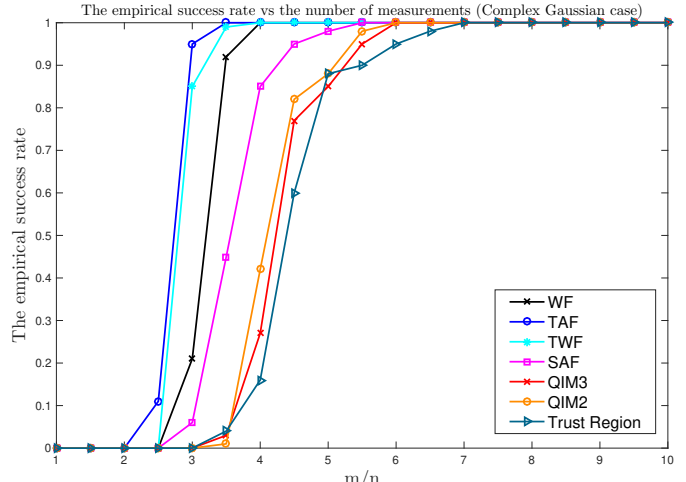

(b)

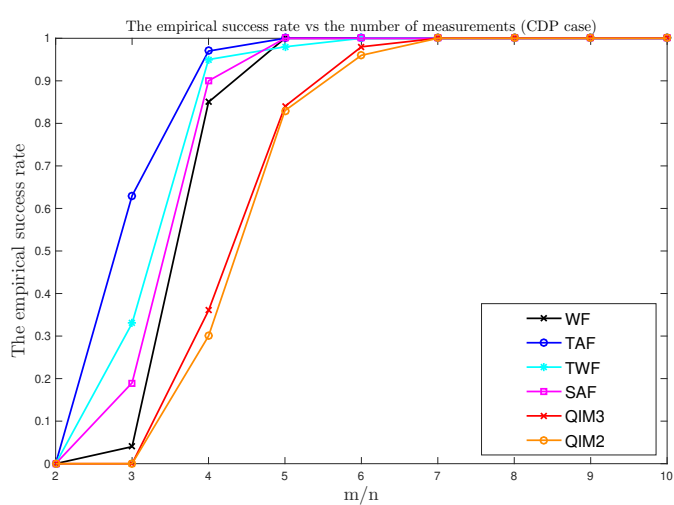

(c)

Figure 1: The empirical success rate for different $m / n$ based on 100 random trails. (a) Success rate for real Gaussian case, (b) Success rate for complex Gaussian case, (c) Success rate for CDP case.

Table 1: Time elapsed and iteration number among algorithms on Gaussian signals with $n=1000$.

\begin{tabular}{||c|cc|cc|cc|cc||}
\hline \multirow{2}{*}{ Algorithm } & \multicolumn{4}{|c|}{ Real Gaussian } & \multicolumn{3}{c||}{ Complex Gaussian } \\
\cline { 2 - 10 } & \multicolumn{2}{|c|}{$10^{-5}$} & \multicolumn{2}{|c|}{$10^{-10}$} & \multicolumn{2}{c|}{$10^{-5}$} & \multicolumn{2}{c||}{$10^{-10}$} \\
\hline & Iter & Time(s) & Iter & Time(s) & Iter & Time(s) & Iter & Time(s) \\
\hline SAF & 44 & $\mathbf{0 . 1 5 5 6}$ & 68 & $\mathbf{0 . 2 2 7 6}$ & 113 & $\mathbf{1 . 3 0 9 2}$ & 190 & $\mathbf{2 . 3 5 9 6}$ \\
\hline QIM2 & 58 & 2.0589 & 117 & 3.7204 & 155 & 21.6235 & 314 & 37.1972 \\
\hline QIM3 & 88 & 2.4423 & 161 & 4.2229 & 211 & 30.2235 & 422 & 48.1972 \\
\hline WF & 125 & 4.4214 & 229 & 6.3176 & 304 & 34.6266 & 655 & 86.6993 \\
\hline TAF & 29 & 0.2744 & 60 & 0.3515 & 100 & 1.7704 & 211 & 2.7852 \\
\hline TWF & 40 & 0.3181 & 87 & 0.4274 & 112 & 1.9808 & 244 & 3.7432 \\
\hline Trust Region & $\mathbf{2 1}$ & 2.9832 & $\mathbf{2 9}$ & 4.4683 & $\mathbf{3 3}$ & 19.1252 & $\mathbf{4 2}$ & 29.0338 \\
\hline
\end{tabular}




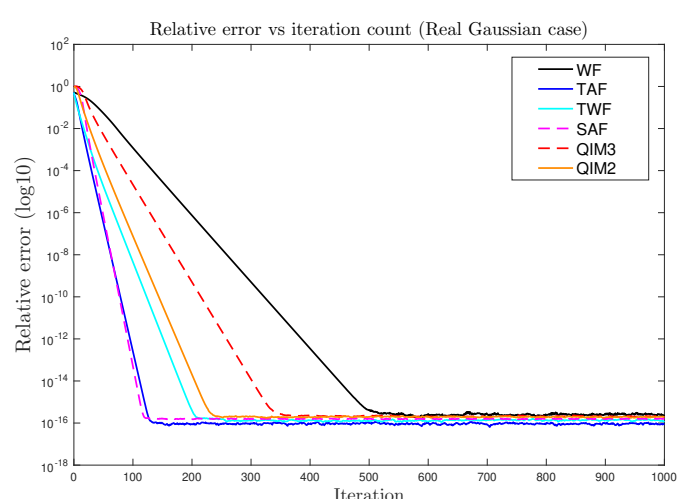

(a)

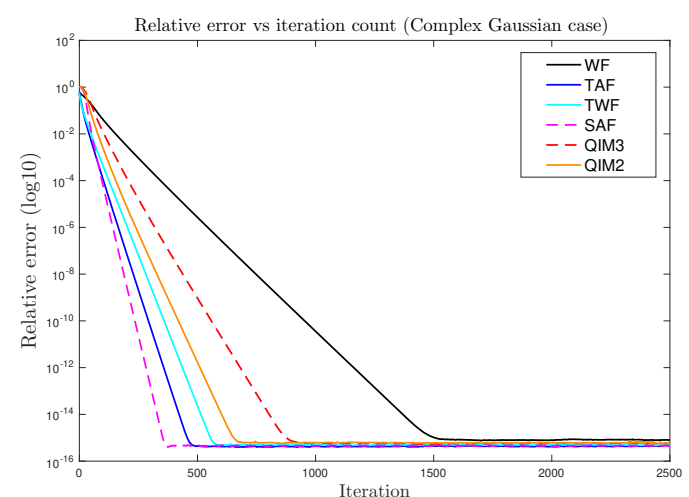

(b)

Figure 2: Relative error versus number of iterations for QIM, SAF, WF, TWF, and TAF method: (a) Real-valued signals; (b) Complex-valued signals.

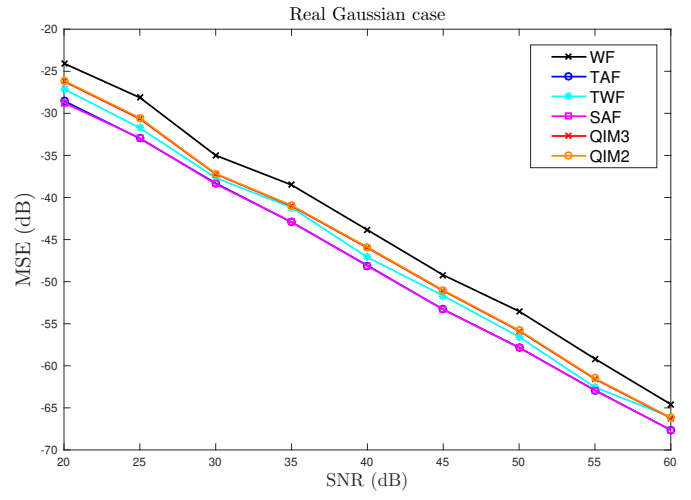

(a)

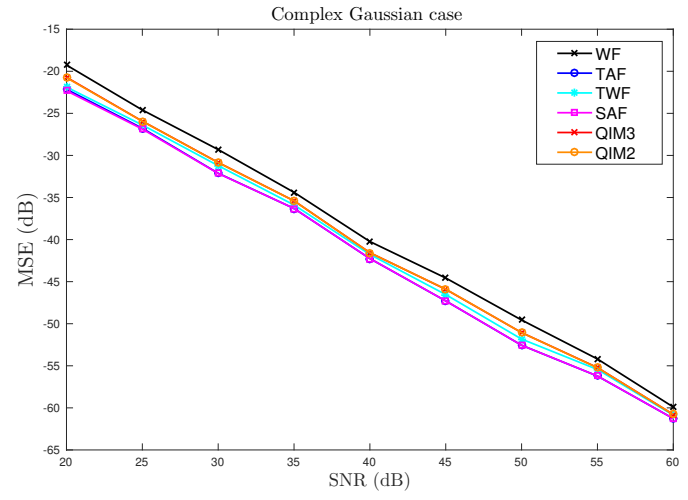

(b)

Figure 3: SNR versus relative MSE on a dB-scale under the noisy Gaussian model: (a) Real Gaussian case; (b) Complex Gaussian case.

\subsection{Recovery of natural image}

We next compare the performance of the above algorithms on recovering a natural image from masked Fourier intensity measurements. The image is the Milky Way Galaxy with resolution $1080 \times 1920$. The colored image has RGB channels. We use $L=20$ random octanary patterns to obtain the Fourier intensity measurements for each $\mathrm{R} / \mathrm{G} / \mathrm{B}$ channel as in [3]. Table 2 lists the averaged time elapsed and the iteration needed to achieve the relative error $10^{-5}$ and $10^{-10}$ over the three RGB channels. We can see that our algorithms have good performance comparing with state-of-the-art algorithms with spectral initialization. 
Table 2: Time elapsed and iteration number among algorithms on recovery of galaxy image.

\begin{tabular}{||c|cc|cc||}
\hline \multirow{2}{*}{ Algorithm } & \multicolumn{3}{|c||}{ The Milky Way Galaxy } \\
\cline { 2 - 5 } & \multicolumn{2}{|c||}{$10^{-5}$} & \multicolumn{2}{c||}{$10^{-10}$} \\
\hline & Iter & Time(s) & Iter & Time(s) \\
\hline SAF & 92 & $\mathbf{2 0 2 . 4 7}$ & 148 & $\mathbf{3 5 1 . 2 1}$ \\
\hline QIM2 & 168 & 351.32 & 282 & 601.68 \\
\hline QIM3 & 173 & 371.59 & 296 & 709.21 \\
\hline WF & 158 & 381.7 & 277 & 621.63 \\
\hline TAF & $\mathbf{6 5}$ & 223.89 & $\mathbf{1 2 2}$ & 368.22 \\
\hline TWF & 68 & 315.14 & 145 & 566.84 \\
\hline
\end{tabular}

\subsection{Recovery of signals with noise}

We now demonstrate the robustness of QIM2, QIM3 to noise and compare them with SAF, WF, TWF, TAF. We consider the noisy model $y_{i}=\left|\left\langle a_{i}, x\right\rangle\right|+\eta_{i}$ and add different level of Gaussian noises to explore the relationship between the signal-tonoise rate (SNR) of the measurements and the mean square error (MSE) of the recovered signal. Specifically, SNR and MSE are evaluated by

$$
\mathrm{MSE}:=10 \log _{10} \frac{\operatorname{dist}^{2}(u, x)}{\|x\|^{2}} \text { and } \mathrm{SNR}=10 \log _{10} \frac{\sum_{i=1}^{m}\left|a_{i}^{\top} x\right|^{2}}{\|\eta\|^{2}},
$$

where $u$ is the output of the algorithms given above after 2500 iterations. We choose $n=128$ and $m=8 n$. The SNR varies from $20 \mathrm{db}$ to $60 \mathrm{db}$. The result is shown in Fig. 3. We can see that our algorithms are stable for noisy phase retrieval.

\section{Appendix A: technical estimates for Section 2}

Lemma A.1. Let $\phi \in C_{c}^{\infty}(\mathbb{R})$ satisfies $0 \leq \phi(x) \leq 1$ for all $x, \phi(x)=1$ for $|x| \leq 1$ and $\phi(x)=0$ for $|x| \geq 2$. There exist $\epsilon>0$ sufficiently small, and $N$ sufficiently large such that

$$
\mathbb{E} \frac{(a \cdot \xi)^{2}\left(a \cdot e_{1}\right)^{2}}{\epsilon+\left(a \cdot e_{1}\right)^{2}} \phi\left(\frac{a \cdot \xi}{N}\right) \geq 0.99, \quad \forall \xi \in \mathbb{S}^{n-1},
$$

where $a \sim \mathcal{N}\left(0, \mathrm{I}_{\mathrm{n}}\right)$.

Proof. We first show that there exist $\epsilon>0$, such that

$$
\mathbb{E} \frac{(a \cdot \xi)^{2}\left(a \cdot e_{1}\right)^{2}}{\epsilon+\left(a \cdot e_{1}\right)^{2}} \geq 0.995, \quad \forall \xi \in \mathbb{S}^{n-1} .
$$


Clearly it suffices for us to show

$$
\sup _{\xi \in \mathbb{S}^{n-1}} \mathbb{E} \frac{\epsilon(a \cdot \xi)^{2}}{\epsilon+\left(a \cdot e_{1}\right)^{2}} \rightarrow 0 \quad \text { as } \epsilon \rightarrow 0
$$

Observe that $\xi=s e_{1}+\sqrt{1-s^{2}} e_{1}^{\perp},|s| \leq 1, e^{\perp} \cdot e_{1}=0$. Thus denoting $X$ and $Y$ as two independent standard Gaussian random variables with mean zero and unit variance, we have

$$
\sup _{\xi \in \mathbb{S}^{n-1}} \mathbb{E} \frac{\epsilon(a \cdot \xi)^{2}}{\epsilon+\left(a \cdot e_{1}\right)^{2}} \lesssim \mathbb{E} \frac{\epsilon X^{2}}{\epsilon+X^{2}}+\mathbb{E} \frac{\epsilon Y^{2}}{\epsilon+X^{2}} \lesssim \epsilon+\mathbb{E} \frac{\epsilon}{\epsilon+X^{2}} \lesssim \sqrt{\epsilon}
$$

where in the last inequality we used the fact that

$$
\int_{|x| \leq 1} \frac{\epsilon}{\epsilon+x^{2}} d x \sim \sqrt{\epsilon}
$$

Thus (A.2) and (A.1) hold. Now $\epsilon$ is fixed. To show the final inequality, we note that

$$
\mathbb{E} \frac{(a \cdot \xi)^{2}\left(a \cdot e_{1}\right)^{2}}{\epsilon+\left(a \cdot e_{1}\right)^{2}} \chi_{|a \cdot \xi| \geq N} \leq \mathbb{E}(a \cdot \xi)^{2} \chi_{|a \cdot \xi| \geq N} \leq \mathbb{E} X^{2} \chi_{|X| \geq N} \rightarrow 0,
$$

as $N$ tend to infinity. Thus the desired inequality easily follows.

Lemma A.2. Let $0<\eta_{0} \ll 1$ be given. Then if $m \gtrsim n$, then the following hold with high probability:

$$
\frac{1}{m} \sum_{k=1}^{m} \frac{\left(a_{k} \cdot \hat{u}\right)^{4}}{\left(a_{k} \cdot e_{1}\right)^{2}} \geq 100, \quad \forall \hat{u} \in \mathbb{S}^{n-1} \quad \text { with }|| \hat{u} \cdot e_{1}|-1| \geq \eta_{0} .
$$

Proof. Without loss of generality we write

$$
\hat{u}=s e^{\perp} \pm \sqrt{1-s^{2}} e_{1}, \quad e^{\perp} \in \mathbb{S}^{n-1} \quad \text { with } \quad e^{\perp} \cdot e_{1}=0 .
$$

Clearly $|s| \geq s_{0}=s_{0}\left(\eta_{0}\right)>0$, where $s_{0}\left(\eta_{0}\right)$ is a constant depending only on $\eta_{0}$. Take $a \sim \mathcal{N}\left(0, \mathrm{I}_{\mathrm{n}}\right)$ and observe that

$$
\begin{aligned}
& \mathbb{E} \frac{(a \cdot \hat{u})^{4}}{\epsilon\left(1+\left(a \cdot e^{\perp}\right)^{2}\right)+\left(a \cdot e_{1}\right)^{2}} \\
\geq & \mathbb{E} \frac{s^{4}\left(a \cdot e^{\perp}\right)^{4}}{\epsilon\left(1+\left(a \cdot e^{\perp}\right)^{2}\right)+\left(a \cdot e_{1}\right)^{2}} \\
\geq & s_{0}^{4} \frac{1}{2 \pi} \int_{1 \leq y \leq 2, x \in \mathbb{R}} \frac{y^{4}}{\epsilon\left(1+y^{2}\right)+x^{2}} e^{-\frac{x^{2}+y^{2}}{2}} d x d y \\
\geq & s_{0}^{4} \frac{1}{200} \int_{|x| \leq 1} \frac{1}{5 \epsilon+x^{2}} d x \geq s_{0}^{4} \cdot \mathcal{O}\left(\epsilon^{-\frac{1}{2}}\right) \geq 200,
\end{aligned}
$$


if $\epsilon>0$ is taken sufficiently small. Now we fix this $\epsilon$. Clearly for $m \gtrsim n$ with high probability it holds that

$$
\begin{aligned}
\frac{1}{m} \sum_{k=1}^{m} \frac{\left(a_{k} \cdot \hat{u}\right)^{4}}{\left(a_{k} \cdot e_{1}\right)^{2}} & \geq \frac{1}{m} \sum_{k=1}^{m} \frac{\left(a_{k} \cdot \hat{u}\right)^{4}}{\epsilon\left(1+\left(a_{k} \cdot e^{\perp}\right)^{2}\right)+\left(a_{k} \cdot e_{1}\right)^{2}} \\
& \geq 100, \quad \forall \hat{u} \in \mathbb{S}^{n-1} \quad \text { with }|| \hat{u} \cdot e_{1}|-1| \leq \eta_{0} .
\end{aligned}
$$

Thus, we complete the proof.

\section{Appendix B: technical estimates for Section 3}

Lemma B.1. For any $\epsilon>0$, there exists $R_{0}=R_{0}(\beta, \epsilon)>0$ sufficiently small, such that if $m \gtrsim n$, then the following hold with high probability:

$$
\frac{R}{m} \sum_{k=1}^{m} \frac{\left(a_{k} \cdot \hat{u}\right)^{2}}{\beta R+\left(a_{k} \cdot e_{1}\right)^{2}}<\epsilon, \quad \forall \hat{u} \in \mathbb{S}^{n-1}, \quad \forall 0<R \leq R_{0} .
$$

Proof. Let $\phi \in C_{c}^{\infty}(\mathbb{R})$ be such that $0 \leq \phi(x) \leq 1$ for all $x, \phi(x)=1$ for $|x| \leq 1$ and $\phi(x)=0$ for $|x| \geq 2$. We then split the sum as

$$
\frac{R}{m} \sum_{k=1}^{m} \frac{\left(a_{k} \cdot \hat{u}\right)^{2}}{\beta R+\left(a_{k} \cdot e_{1}\right)^{2}} \leq \frac{1}{\beta m} \sum_{k=1}^{m}\left(a_{k} \cdot \hat{u}\right)^{2} \phi\left(\frac{a_{k} \cdot e_{1}}{\eta_{0}}\right)+R \cdot \eta_{0}^{-2} \frac{1}{m} \sum_{k=1}^{m}\left(a_{k} \cdot \hat{u}\right)^{2} .
$$

Clearly the first term is amenable to union bounds, and we can make it sufficiently small with high probability by taking $\eta_{0}$ small (depending only on $\beta$ and $\epsilon$ ). The second term is trivial since we can take $R$ sufficiently small. Thus we complete the proof.

Lemma B.2. There exists $R_{1}=R_{1}(\beta)>0$ sufficiently small, such that if $m \gtrsim n$, then the following hold with high probability:

$$
c_{1} \leq \frac{1}{m} \sum_{k=1}^{m} \frac{\left(a_{k} \cdot \hat{u}\right)\left(a_{k} \cdot e_{1}\right)^{3}}{\beta R+\left(a_{k} \cdot e_{1}\right)^{2}} \leq c_{2}, \quad \forall \hat{u} \in \mathbb{S}^{n-1} \quad \text { with } \widehat{u}_{1} \cdot e_{1} \geq \frac{1}{10}, \quad \forall 0<R \leq R_{1} .
$$

In the above $c_{1}, c_{2}>0$ are constants depending only on $\beta$.

Proof. Denote $X_{k}=a_{k} \cdot e_{1}$. Write $\hat{u}=s e_{1}+\sqrt{1-s^{2}} e^{\perp}$, where $s \geq \frac{1}{10}$ and $e^{\perp} \in \mathbb{S}^{n-1}$ 
satisfies $e^{\perp} \cdot e_{1}=0$. We then write

$$
\begin{aligned}
& \frac{1}{m} \sum_{k=1}^{m} \frac{\left(a_{k} \cdot \hat{u}\right) X_{k}^{3}}{\beta R+X_{k}^{2}} \\
= & s \frac{1}{m} \sum_{k=1}^{m} \frac{X_{k}^{4}}{\beta R+X_{k}^{2}}+\sqrt{1-s^{2}} \frac{1}{m} \sum_{k=1}^{m} \frac{\left(a_{k} \cdot e^{\perp}\right) X_{k}^{3}}{\beta R+X_{k}^{2}} \\
= & s \frac{1}{m} \sum_{k=1}^{m} \frac{X_{k}^{4}}{\beta R+X_{k}^{2}}+\sqrt{1-s^{2}} \frac{1}{m} \sum_{k=1}^{m}\left(a_{k} \cdot e^{\perp}\right) X_{k}-\sqrt{1-s^{2}} \frac{1}{m} \sum_{k=1}^{m}\left(a_{k} \cdot e^{\perp}\right) \frac{\beta R X_{k}}{\beta R+X_{k}^{2}} .
\end{aligned}
$$

For the first term we note that for $0<R \leq 1$,

$$
\frac{1}{m} \sum_{k=1}^{m} \frac{X_{k}^{4}}{\beta+X_{k}^{2}} \leq \frac{1}{m} \sum_{k=1}^{m} \frac{X_{k}^{4}}{\beta R+X_{k}^{2}} \leq \frac{1}{m} \sum_{k=1}^{m} X_{k}^{2} .
$$

Thus we clearly have for all $0<R \leq 1, \frac{1}{10} \leq s \leq 1$, with high probability it holds that

$$
2 c_{1} \leq s \frac{1}{m} \sum_{k=1}^{m} \frac{X_{k}^{4}}{\beta R+X_{k}^{2}} \leq \frac{1}{2} c_{2} .
$$

The second term is clearly OK for union bounds and with high probability it can be made sufficiently small. For the last term, observe that with high probability,

$$
\frac{1}{m} \sum_{k=1}^{m}\left|a_{k} \cdot e^{\perp}\right| \frac{\beta R\left|X_{k}\right|}{\beta R+X_{k}^{2}} \lesssim \sqrt{\beta R} \frac{1}{m} \sum_{k=1}^{m}\left|a_{k} \cdot e^{\perp}\right| \ll 1, \quad \forall e^{\perp} \in \mathbb{S}^{n-1},
$$

if $R \leq R_{1}$ and $R_{1}$ is sufficiently small. The desired result then clearly follows.

Proof of Lemma 3.5. Without loss of generality we consider the situation $\hat{u}=e_{1} \cos \theta+$ $e^{\perp} \sin \theta$ with $\epsilon_{1} \leq \theta \leq \frac{\pi}{2}-\epsilon_{2}$, where $0<\epsilon_{1}, \epsilon_{2} \ll 1$. The point is that $\theta$ stays away from the end-points 0 and $\frac{\pi}{2}$. Denote $X_{k}=a_{k} \cdot e_{1}, Y_{k}=a_{k} \cdot e^{\perp}$ and $Z_{k}=a_{k} \cdot \hat{u}$. Then

$$
\begin{aligned}
& Z_{k}=\cos \theta X_{k}+\sin \theta Y_{k} \Rightarrow Y_{k}=\frac{1}{\sin \theta} Z_{k}-\frac{\cos \theta}{\sin \theta} X_{k} ; \\
& \partial_{\theta} Z_{k}=-\sin \theta X_{k}+\cos \theta Y_{k}=\cot \theta Z_{k}-\frac{1}{\sin \theta} X_{k} .
\end{aligned}
$$

We then obtain

$$
\begin{aligned}
& \partial_{\theta} f= 4 R^{2} \cot \theta \\
& \underbrace{\frac{1}{m} \sum_{k=1}^{m} \frac{Z_{k}^{4}}{\beta R+X_{k}^{2}}}_{=: H_{0}}-4 R^{2} \csc \theta \frac{1}{m} \sum_{k=1}^{m} \frac{Z_{k}^{3} X_{k}}{\beta R+X_{k}^{2}} \\
&-4 R \cot \theta \frac{1}{m} \sum_{k=1}^{m} \frac{Z_{k}^{2} X_{k}^{2}}{\beta R+X_{k}^{2}}+4 R \csc \theta \frac{1}{m} \sum_{k=1}^{m} \frac{Z_{k} X_{k}^{3}}{\beta R+X_{k}^{2}} .
\end{aligned}
$$


Since $R \sim 1$, it is not difficult to check that the third and fourth terms above are amenable to union bounds ${ }^{\dagger}$, i.e., with high probability (for $m \gtrsim n$ ) we have

$$
\mid \frac{1}{m} \sum_{k=1}^{m} \frac{Z_{k}^{2} X_{k}^{2}}{\beta R+X_{k}^{2}}-\text { mean }|+| \frac{1}{m} \sum_{k=1}^{m} \frac{Z_{k} X_{k}^{3}}{\beta R+X_{k}^{2}}-\text { mean } \mid \ll 1, \quad \forall c_{1} \leq R \leq c_{2}, \quad \forall \hat{u} \in \mathbb{S}^{n-1} .
$$

Next we treat the second term. Let $\phi \in C_{c}^{\infty}(\mathbb{R})$ be such that $0 \leq \phi(x) \leq 1$ for all $x$, $\phi(x)=1$ for $|x| \leq 1$ and $\phi(x)=0$ for $|x| \geq 2$. We have

$$
\frac{1}{m} \sum_{k=1}^{m} \frac{Z_{k}^{3} X_{k}}{\beta R+X_{k}^{2}}=\underbrace{\frac{1}{m} \sum_{k=1}^{m} \frac{Z_{k}^{3} X_{k}}{\beta R+X_{k}^{2}} \phi\left(\frac{Z_{k}}{M\left\langle X_{k}\right\rangle}\right)}_{=: H_{1}}+\underbrace{\frac{1}{m} \sum_{k=1}^{m} \frac{Z_{k}^{3} X_{k}}{\beta R+X_{k}^{2}}\left(1-\phi\left(\frac{Z_{k}}{M\left\langle X_{k}\right\rangle}\right)\right)}_{=: H_{2}},
$$

where $\langle z\rangle=\left(1+|z|^{2}\right)^{\frac{1}{2}}$. It is not difficult to check that $H_{1}$ is OK for union bounds, and with high probability it holds that

$$
\left|H_{1}-\mathbb{E} H_{1}\right| \ll 1, \quad \forall \hat{u} \in \mathbb{S}^{n-1}, \quad \forall c_{1} \leq R \leq c_{2} .
$$

For $H_{2}$ we have ( $\eta_{0}$ will be taken sufficiently small)

$$
\begin{aligned}
H_{2} & \leq \eta_{0} \frac{1}{m} \sum_{k=1}^{m} \frac{Z_{k}^{4}}{\beta R+X_{k}^{2}}+\eta_{0}^{-3} \frac{1}{m} \sum_{k=1}^{m} \frac{X_{k}^{4}}{\beta R+X_{k}^{2}}\left(1-\phi\left(\frac{Z_{k}}{M\left\langle X_{k}\right\rangle}\right)\right) \\
& \leq \underbrace{\eta_{0} \frac{1}{m} \sum_{k=1}^{m} \frac{Z_{k}^{4}}{\beta R+X_{k}^{2}}}_{=: H_{2, a}}+\underbrace{\eta_{0}^{-3} \frac{1}{m} \sum_{k=1}^{m} X_{k}^{2}\left(1-\phi\left(\frac{Z_{k}}{M\left\langle X_{k}\right\rangle}\right)\right)}_{=: H_{2, b}} .
\end{aligned}
$$

We first take $\eta_{0}$ sufficiently small so that $H_{2, a}$ can be included in the estimate of $H_{0}$ without affecting too much the main order. On the other hand, once $\eta_{0}$ is fixed, we can take $M$ sufficiently large such that

$$
\left|H_{2, b}\right|+\left|\mathbb{E} H_{2, b}\right| \ll 1, \quad \forall \hat{u} \in \mathbb{S}^{n-1}, \quad \forall c_{1} \leq R \leq c_{2} .
$$

Finally we treat $H_{0}$. Clearly

$$
H_{0} \geq \underbrace{\frac{1}{m} \sum_{k=1}^{m} \frac{Z_{k}^{4}}{\beta R+X_{k}^{2}} \phi\left(\frac{Z_{k}}{K}\right)}_{=: H_{0, a}} .
$$

${ }^{\dagger}$ The union bound includes covering in $\hat{u}$ and $R$. 
By taking $K$ large, it can be easily checked that

$$
\sup _{\hat{u} \in \mathbb{S}^{n-1}, c_{1} \leq R \leq c_{2}}\left|\mathbb{E} H_{0}-\mathbb{E} H_{0, a}\right| \ll 1 .
$$

On the other hand, for fixed $K$, clearly $H_{0, a}$ is OK for union bounds. It holds with high probability that

$$
\left|H_{0, a}-\mathbb{E} H_{0, a}\right| \ll 1
$$

Collecting all the estimates, we obtain

$$
\partial_{\theta} f \geq \mathbb{E} \partial_{\theta} f+\text { Error }
$$

where $\mid$ Error $\mid \ll 1$. The desired lower bound for $\partial_{\theta} f$ then easily follows from Lemma B.3 below.

Lemma B.3. Let $u=\sqrt{R} \hat{u}$ with $0<c_{1} \leq R \leq c_{2}<\infty$ and $\hat{u} \in \mathbb{S}^{n-1}$. Assume $\hat{u}=$ $\cos \theta e_{1}+\sin \theta e^{\perp}$, where $\theta \in[0, \pi]$ and $e^{\perp} \in \mathbb{S}^{n-1}$ satisfies $e^{\perp} \cdot e_{1}=0$. We have

$$
\mathbb{E} f(u)=h\left(\beta, R, \cos ^{2} \theta\right),
$$

where

$$
\begin{gathered}
\max _{0 \leq s \leq 1} \partial_{s} h(\beta, R, s) \leq-\gamma_{1}<0, \\
\min _{0 \leq s \leq 1} \partial_{s s} h(\beta, R, s) \geq \gamma_{2}>0 .
\end{gathered}
$$

Here $\gamma_{i}=\gamma_{i}\left(\beta, c_{1}, c_{2}\right), i=1,2$ depend only on $\left(\beta, c_{1}, c_{2}\right)$. It follows that

$$
\begin{aligned}
& \mathbb{E} \partial_{\theta} f=a_{1}\left(\beta, R, \cos ^{2} \theta\right) \sin (2 \theta) ; \\
& \mathbb{E} \partial_{\theta \theta} f=2 a_{1}\left(\beta, R, \cos ^{2} \theta\right) \cos (2 \theta)+a_{2}(\beta, R, \theta) \sin ^{2}(2 \theta),
\end{aligned}
$$

where

$$
\gamma_{3}<a_{i}(\beta, R, s) \leq \gamma_{4}, \quad \forall s \in[0,1], \quad i=1,2 ;
$$

and $\gamma_{3}>0, \gamma_{4}>0$ are constants depending only on $\left(\beta, c_{1}, c_{2}\right)$.

Proof. We have

$$
\begin{aligned}
\mathbb{E} f(u) & =\frac{1}{2 \pi} \int_{\mathbb{R}^{2}} \frac{\left(R(x \cos \theta+y \sin \theta)^{2}-x^{2}\right)^{2}}{\beta R+x^{2}} e^{-\frac{x^{2}+y^{2}}{2}} d x d y \\
& =\frac{1}{\pi} \int_{0}^{\infty} \frac{1}{\beta R+x^{2}} e^{-\frac{x^{2}}{2}} \cdot \sqrt{2 \pi} h_{1}\left(R, x, \cos ^{2} \theta\right) d x,
\end{aligned}
$$


where

$$
h_{1}(R, x, s)=3 R^{2}-2 R x^{2}+x^{4}+s\left(6 R^{2}-2 R x^{2}\right)\left(-1+x^{2}\right)+R^{2} s^{2}\left(3-6 x^{2}+x^{4}\right) .
$$

Integrating further in $x$ then gives

$$
\mathbb{E} f(u)=\sqrt{2 \pi} \cdot \frac{1}{\pi} \cdot R\left(c_{1} s^{2}+2 c_{2} s+c_{3}\right), \quad s=\cos ^{2} \theta,
$$

where the value of $c_{3}$ is unimportant for us, and

$$
\begin{aligned}
& c_{1}=R \int_{0}^{\infty} \frac{1}{\beta R+x^{2}} e^{-\frac{x^{2}}{2}}\left(3-6 x^{2}+x^{4}\right) d x ; \\
& c_{2}=\int_{0}^{\infty} \frac{1}{\beta R+x^{2}} e^{-\frac{x^{2}}{2}}\left(3 R-x^{2}\right)\left(-1+x^{2}\right) d x .
\end{aligned}
$$

First we show that $c_{2}<0$. By a short computation, we have

$$
c_{2}=\frac{3+\beta}{2 \beta} \cdot\left(\beta R \sqrt{2 \pi}-e^{\frac{\beta R}{2}} \pi \sqrt{\beta R}(1+\beta R) \operatorname{Erfc}\left(\sqrt{\frac{\beta R}{2}}\right)\right),
$$

where

$$
\operatorname{Erfc}(y)=\frac{2}{\sqrt{\pi}} \int_{y}^{\infty} e^{-t^{2}} d t
$$

We then reduce the matter to showing

$$
y<e^{y^{2}}\left(1+2 y^{2}\right) \int_{y}^{\infty} e^{-t^{2}} d t, \quad \forall y>0 .
$$

This follows easily from the usual bound on $\operatorname{Erfc}(y)$ :

$$
\frac{1}{y+\sqrt{y^{2}+2}}<\operatorname{Erfc}(y) \cdot e^{y^{2}} \cdot \frac{\sqrt{\pi}}{2} \leq \frac{1}{y+\sqrt{y^{2}+\frac{4}{\pi}}}, \quad \forall y>0 .
$$

Thus $c_{2}<0$.

Next we show that $c_{1}>0$. We have

$$
2 \beta c_{1}=-\sqrt{2 \pi} \beta R(5+\beta R)+e^{\frac{\beta R}{2}} \pi \sqrt{\beta R}(3+\beta R(6+\beta R)) \cdot \operatorname{Erfc}\left(\sqrt{\frac{\beta R}{2}}\right) .
$$

It amounts to checking

$$
e^{y^{2}} \int_{y}^{\infty} e^{-t^{2}} d t>\frac{y\left(5+2 y^{2}\right)}{3+4 y^{2}\left(3+y^{2}\right)}, \quad \forall y>0 .
$$


This follows from Lemma B.4 below.

Finally we show $c_{1}+c_{2}<0$. We have

$$
\begin{aligned}
& 2\left(c_{1}+c_{2}\right) \\
= & \sqrt{2 \pi} R(-2+\beta-\beta R)-e^{\frac{\beta R}{2}} \pi \cdot\left(-\beta^{\frac{3}{2}} R^{\frac{5}{2}}+(\beta R)^{\frac{3}{2}}+\sqrt{\beta R}-3 R \sqrt{\beta R}\right) \operatorname{Erfc}\left(\sqrt{\frac{\beta R}{2}}\right) .
\end{aligned}
$$

Denote $y=\sqrt{\frac{\beta R}{2}}>0$. We then reduce matters to showing

$$
2 y^{2}-2 R\left(1+y^{2}\right)<e^{y^{2}} \cdot 2 y \cdot\left(-2 y^{2} R-3 R+1+2 y^{2}\right) \int_{y}^{\infty} e^{-t^{2}} d t .
$$

Since we have shown (B.1), we then only need to check

$$
1+y^{2}>e^{y^{2}} y\left(2 y^{2}+3\right) \int_{y}^{\infty} e^{-t^{2}} d t
$$

This in turn follows from Lemma B.4.

Finally we consider the polynomial

$$
\tilde{h}(s)=c_{1} s^{2}+2 c_{2} s .
$$

Since $\tilde{h}^{\prime}(s)=2 c_{1} s+2 c_{2}$ and $\tilde{h}^{\prime}(0)=2 c_{2}<0, \tilde{h}^{\prime}(1)=2 c_{1}+2 c_{2}<0$, we have $\tilde{h}^{\prime}(s)<0$ for all $s \in[0,1]$. Since $c_{1}>0$, we have $\tilde{h}^{\prime \prime}(s)>0$. The desired result then easily follows.

Lemma B.4 (Refined upper and lower bounds on the Complementary Error function). Let

$$
\operatorname{Erfc}(x)=\frac{2}{\sqrt{\pi}} \int_{x}^{\infty} e^{-t^{2}} d t \quad \text { for } x>0
$$

Then

$$
\begin{array}{ll}
e^{x^{2}} \cdot \operatorname{Erfc}(x) \cdot \frac{\sqrt{\pi}}{2}>\frac{x\left(5+2 x^{2}\right)}{3+4 x^{2}\left(3+x^{2}\right)}, & \forall x>0 ; \\
e^{x^{2}} \cdot \operatorname{Erfc}(x) \cdot \frac{\sqrt{\pi}}{2}<\frac{1+x^{2}}{x\left(3+2 x^{2}\right)}, & \forall x>0 .
\end{array}
$$

Remark B.1. In the regime $y \geq 1$, one can check that the upper and lower bounds here are sharper than (B.2). One should also recall that the usual way to derive the lower bound in (B.2) through conditional expectation. Namely one can regard $e^{-y^{2}} /(\sqrt{\pi} \operatorname{Erfc}(y))$ as the conditional mean $\mu_{1}(y)=\mathbb{E}(X \mid X>y)$ where $X$ has the p.d.f. $\frac{1}{\sqrt{\pi}} e^{-x^{2}}$. Then evaluating the variance $\mathbb{E}\left(\left(X-\mu_{1}\right)^{2} \mid X>y\right)>0$ gives $y \mu_{1}+\frac{1}{2}-\mu_{1}^{2}>0$. This yields the upper bound for $\mu_{1}$ which in turn is the desired lower bound in (B.2). An interesting question is to derive a sharper two-sided bounds via more careful conditioning. However we shall not dwell on this issue here. 
Proof of Lemma B.4. We focus on the regime $x>1$. By performing successive simple change of variables, we have

$$
\begin{aligned}
g(x) & :=e^{x^{2}} \int_{x}^{\infty} e^{-t^{2}} d t=\int_{0}^{\infty} e^{-2 x s} e^{-s^{2}} d s=\frac{1}{2 x} \int_{0}^{\infty} e^{-s} e^{-\left(\frac{s}{2 x}\right)^{2}} d s \\
& \sim \sum_{k=0}^{\infty}(-1)^{k} x^{-(2 k+1)} \cdot \frac{1}{2} \cdot \frac{(2 k) !}{4^{k} k !} \sim \sum_{k=0}^{\infty}(-1)^{k} x^{-(2 k+1)} \cdot \frac{1}{2} \cdot\left(\frac{1}{2}\right)_{k},
\end{aligned}
$$

where in the last line we adopted Pochhammer's symbol $(a)_{n}=a(a+1) \cdots(a+n-1)$. Note that the above is an asymptotic series, and it is not difficult to check that

$$
\left|g(x)-\sum_{k=0}^{m}(-1)^{k} x^{-(2 k+1)} \cdot \frac{1}{2} \cdot\left(\frac{1}{2}\right)_{k}\right| \leq x^{-2 m-3} \cdot \frac{1}{2} \cdot\left(\frac{1}{2}\right)_{m+1}, \quad \forall m \geq 1, \quad \forall x>0 .
$$

Moreover, if $m$ is an even integer, then

$$
g(x)<\sum_{k=0}^{m}(-1)^{k} x^{-(2 k+1)} \cdot \frac{1}{2} \cdot\left(\frac{1}{2}\right)_{k}, \quad \forall x>0 ;
$$

and if $m$ is odd, then

$$
g(x)>\sum_{k=0}^{m}(-1)^{k} x^{-(2 k+1)} \cdot \frac{1}{2} \cdot\left(\frac{1}{2}\right)_{k}, \quad \forall x>0 .
$$

Now taking $m=4$, we have

$$
g(x)<\frac{1}{2} x^{-1}-\frac{1}{4} x^{-3}+\frac{3}{8} x^{-5}-\frac{15}{16} x^{-7}+\frac{105}{32} x^{-9} .
$$

For $x \geq 3$, it is not difficult to verify that

$$
\frac{1}{2} x^{-1}-\frac{1}{4} x^{-3}+\frac{3}{8} x^{-5}-\frac{15}{16} x^{-7}+\frac{105}{32} x^{-9}<\frac{1+x^{2}}{x\left(3+2 x^{2}\right)} .
$$

Hence the upper bound is OK for $x \geq 3$.

Next taking $m=5$, we have

$$
g(x)>\frac{1}{2} x^{-1}-\frac{1}{4} x^{-3}+\frac{3}{8} x^{-5}-\frac{15}{16} x^{-7}+\frac{105}{32} x^{-9}-\frac{945}{64} x^{-11} .
$$

It is not difficult to verify that for $x \geq 4$, we have

$$
\frac{1}{2} x^{-1}-\frac{1}{4} x^{-3}+\frac{3}{8} x^{-5}-\frac{15}{16} x^{-7}+\frac{105}{32} x^{-9}-\frac{945}{64} x^{-11}>\frac{x\left(5+2 x^{2}\right)}{3+12 x^{2}+4 x^{4}} .
$$


Hence the lower bound is OK for $x \geq 4$.

Finally for the regime $x \in[0,4]$, we use rigorous numerics to verify the inequality. Since we are on a compact interval, this can be done by a rigorous computation with controllable numerical errors.

Proof of Lemma 3.6. Again denote $X_{k}=a_{k} \cdot e_{1}$ and $Z_{k}=a_{k} \cdot \hat{u}$. Without loss of generality we assume $\theta \in\left[\frac{\pi}{2}-\eta, \frac{\pi}{2}+\eta\right]$ for some sufficiently small $\eta>0$. By a tedious computation, we have

$$
\begin{aligned}
\partial_{\theta \theta} f=4 & R^{2}(1+2 \cos 2 \theta) \csc ^{2} \theta \frac{1}{m} \sum_{k=1}^{m} \frac{Z_{k}^{4}}{\beta R+X_{k}^{2}}-24 R^{2}(\cot \theta \csc \theta) \frac{1}{m} \sum_{k=1}^{m} \frac{X_{k} Z_{k}^{3}}{\beta R+X_{k}^{2}} \\
& +4 R\left(\csc ^{2} \theta\right)(3 R-\cos 2 \theta) \frac{1}{m} \sum_{k=1}^{m} \frac{Z_{k}^{2} X_{k}^{2}}{\beta R+X_{k}^{2}}+8 R(\cot \theta \csc \theta) \frac{1}{m} \sum_{k=1}^{m} \frac{X_{k}^{3} Z_{k}}{\beta R+X_{k}^{2}} \\
& -4 R \csc ^{2} \theta \frac{1}{m} \sum_{k=1}^{m} \frac{X_{k}^{4}}{\beta R+X_{k}^{2}} .
\end{aligned}
$$

Note that the third, fourth and fifth terms are OK for union bounds. The second and the first term can be handled in a similar way as in the proof of Lemma 3.5. The only difference is that the sign is now negative in the regime $\theta \rightarrow \frac{\pi}{2}$. Using Lemma B.3 it follows that $\partial_{\theta \theta} f<0$ in this regime. We omit the repetitive details.

Proof of Theorem 3.4. Without loss of generality we consider the regime $\left\|u-e_{1}\right\|_{2} \ll$ 1. Before we work out the needed estimates for the restricted convexity, we explain the main difficulty in connection with the full Hessian matrix. Denote $X_{k}=a_{k} \cdot e_{1}$. Then for any $\xi \in \mathbb{S}^{n-1}$, we have

$$
\begin{aligned}
H_{\xi \xi}=\sum_{i, j} \xi_{i} \xi_{j}\left(\partial_{u_{i} u_{j}} f\right)(u) & \\
=12 & \frac{1}{m} \sum_{k=1}^{m} \frac{\left(a_{k} \cdot \xi\right)^{2}\left(a_{k} \cdot u\right)^{2}}{\beta\|u\|_{2}^{2}+X_{k}^{2}} \\
& -4 \frac{1}{m} \sum_{k=1}^{m} \frac{\left(a_{k} \cdot \xi\right)^{2} X_{k}^{2}}{\beta\|u\|_{2}^{2}+X_{k}^{2}} \\
& -16 \beta \frac{1}{m} \sum_{k=1}^{m} \frac{\left(a_{k} \cdot u\right)^{3}\left(a_{k} \cdot \xi\right)(u \cdot \xi)}{\left(\beta\|u\|_{2}^{2}+X_{k}^{2}\right)^{2}} \\
& +16 \beta \frac{1}{m} \sum_{k=1}^{m} \frac{X_{k}^{2}\left(a_{k} \cdot u\right)\left(a_{k} \cdot \xi\right)(u \cdot \xi)}{\left(\beta\|u\|_{2}^{2}+X_{k}^{2}\right)^{2}}
\end{aligned}
$$




$$
\begin{aligned}
& -2 \beta \frac{1}{m} \sum_{k=1}^{m} \frac{\left(\left(a_{k} \cdot u\right)^{2}-X_{k}^{2}\right)^{2}}{\left(\beta\|u\|_{2}^{2}+X_{k}^{2}\right)^{2}} \\
& +8 \beta^{2}(\xi \cdot u)^{2} \frac{1}{m} \sum_{k=1}^{m} \frac{\left(\left(a_{k} \cdot u\right)^{2}-X_{k}^{2}\right)^{2}}{\left(\beta\|u\|_{2}^{2}+X_{k}^{2}\right)^{3}} .
\end{aligned}
$$

First observe that if $u=e_{1}$, then the Hessian can be controlled rather easily thanks to the damping $\beta\|u\|_{2}^{2}+X_{k}^{2}$.

On the other hand, for $u \neq e_{1}$, as far as the lower bound is concerned, the main difficult terms are (B.7) and (B.5) which are out of control if we do not impose any condition on $\xi$ (i.e., using (B.3) to control it). On the other hand, if we restrict $\xi$ to the direction $u-e_{1}$, then we can control these difficult terms by using the main good term (B.3). Namely, introduce the decomposition

$$
u=e_{1}+t \xi
$$

where $t=\left\|u-e_{1}\right\|_{2} \ll 1$. Then for (B.5) we write

$$
\left(a_{k} \cdot u\right)^{3}\left(a_{k} \cdot \xi\right)=\left(a_{k} \cdot u\right)^{2}\left(a_{k} \cdot e_{1}\right)\left(a_{k} \cdot \xi\right)+t\left(a_{k} \cdot u\right)^{2}\left(a_{k} \cdot \xi\right)^{2} .
$$

Since $t \ll 1$, the term $t\left(a_{k} \cdot u\right)^{2}\left(a_{k} \cdot e_{1}\right)^{2}$ (together with the pre-factor term in (B.5)) can be included into (B.3) which still has a good lower bound by using localization. On the other hand, the term $\left(a_{k} \cdot u\right)^{2}\left(a_{k} \cdot e_{1}\right)\left(a_{k} \cdot \xi\right)$ can be split as

$$
\begin{aligned}
& \left(a_{k} \cdot u\right)^{2}\left(a_{k} \cdot e_{1}\right)\left(a_{k} \cdot \xi\right) \\
= & \left(a_{k} \cdot u\right)^{2}\left(a_{k} \cdot e_{1}\right)\left(a_{k} \cdot \xi\right) \phi\left(\frac{a_{k} \cdot u}{K}\right) \\
& +\left(a_{k} \cdot u\right)^{2}\left(a_{k} \cdot e_{1}\right)\left(a_{k} \cdot \xi\right)\left(1-\phi\left(\frac{a_{k} \cdot u}{K}\right)\right),
\end{aligned}
$$

where $\phi$ is a smooth cut-off function satisfying $0 \leq \phi(z) \leq 1$ for all $z \in \mathbb{R}, \phi(z)=1$ for $|z| \leq 1$ and $\phi(z)=0$ for $|z| \geq 2$. Clearly the contribution of (B.9) in (B.5) is OK for union bounds. On the other hand, for (B.10) we have

$$
\begin{aligned}
& \left(a_{k} \cdot u\right)^{2}\left|a_{k} \cdot e_{1}\right|\left|a_{k} \cdot \xi\right| \cdot\left(1-\phi\left(\frac{a_{k} \cdot u}{K}\right)\right) \\
\leq & \left(a_{k} \cdot u\right)^{2} \epsilon\left(a_{k} \cdot \xi\right)^{2}+\epsilon^{-1}\left(a_{k} \cdot u\right)^{2}\left(a_{k} \cdot e_{1}\right)^{2}\left(1-\phi\left(\frac{a_{k} \cdot u}{K}\right)\right) .
\end{aligned}
$$

Clearly this is under control (the first term can again be controlled using (B.3)).

Now we turn to (B.7). The main term is $\left(a_{k} \cdot u\right)^{4}$. We write

$$
\left(a_{k} \cdot u\right)^{2}\left(a_{k} \cdot u\right)^{2}=\left(a_{k} \cdot u\right)^{2}\left(a_{k} \cdot e_{1}\right)^{2}+t^{2}\left(a_{k} \cdot u\right)^{2}\left(a_{k} \cdot \xi\right)^{2}+2 t\left(a_{k} \cdot u\right)^{2}\left(a_{k} \cdot e_{1}\right)\left(a_{k} \cdot \xi\right) .
$$


Clearly then this is also under control.

By further using localization, we can then show that with high probability, it holds that

$$
H_{\xi \xi} \geq \mathbb{E} H_{\xi \xi}+\text { Error }
$$

where $\mid$ Error $\mid \ll 1$. The desired conclusion then follows from Lemma B.5.

Remark B.2. Introduce the parametrization $u=\sqrt{R}\left(e_{1} \cos \theta+e^{\perp} \sin \theta\right)$, where $e^{\perp} \in$ $e_{1}=0,|R-1| \ll 1$ and $|\theta| \ll 1$. One might hope to prove that the Hessian matrix

$$
\left(\begin{array}{cc}
\partial_{R R} f & \partial_{R \theta} f \\
\partial_{R \theta} f & \partial_{\theta \theta} f
\end{array}\right)
$$

is positive definite near $u=e_{1}$ under the mere assume $m \gtrsim n$ and with high probability. However there is a subtle issue which we explain as follows. Consider the main term (write $X=a_{k} \cdot e_{1}$ and $Y=a_{k} \cdot e^{\perp}$ )

$$
\tilde{f}=\tilde{f}_{k}=\frac{\left(R(X \cos \theta+Y \sin \theta)^{2}-X^{2}\right)^{2}}{\beta R+X^{2}} .
$$

The most troublesome piece come from quartic and cubic terms in $Y$, and we consider

$$
\tilde{h}_{1}=\frac{R^{2} Y^{4} \sin ^{4} \theta}{\beta R+X^{2}}, \quad \tilde{h}_{2}=\frac{R^{2}\left(4 Y^{3} X \sin ^{3} \theta \cos \theta\right)}{\beta R+X^{2}} .
$$

For $\tilde{h}_{2}$ we do not have a favorable sign and the only hope is to control it via $\tilde{h}_{1}$. On the other hand, for $\tilde{h}_{1}$, we can take $X=Y=1, \beta=1$, and compute

$$
\left(\partial_{R R} \tilde{h}_{1}\right) \cdot\left(\partial_{\theta \theta} \tilde{h}_{1}\right)-\left(\partial_{R \theta} \tilde{h}_{1}\right)^{2}=-\frac{8 R^{2}}{(1+R)^{4}} \sin ^{6} \theta \cdot\left(3+4 R+R^{2}+\left(2+4 R+R^{2}\right) \cos 2 \theta\right) .
$$

In yet other words, the sign is not favorable and this renders the Hessian out of control (before taking the expectation).

Lemma B.5. Let $u=e_{1}+t \xi$, where $\xi \in \mathbb{S}^{n-1}$. Then for $|t| \ll 1$, we have

$$
\mathbb{E} \partial_{t t} f(u) \geq c_{0}>0, \quad \forall \xi \in \mathbb{S}^{n-1},
$$

where $c_{0}>0$ is a constant depending only on $\beta$. 
Proof. Introduce the parametrization $\xi=s e_{1}+\sqrt{1-s^{2}} e^{\perp}$, where $e^{\perp} \cdot e_{1}=0,|s| \leq 1$. Then

$$
u=e_{1}+t\left(s e_{1}+\sqrt{1-s^{2}} e^{\perp}\right)=(1+t s) e_{1}+t \sqrt{1-s^{2}} e^{\perp} .
$$

Thus

$$
\mathbb{E} f(u)=\frac{1}{2 \pi} \int_{\mathbb{R}^{2}} \underbrace{\frac{\left(\left((1+t s) x+t \sqrt{1-s^{2}} y\right)^{2}-x^{2}\right)^{2}}{\beta\left(1+2 t s+t^{2}\right)+x^{2}}}_{=: h(t, s, x, y)} e^{-\frac{x^{2}+y^{2}}{2}} d x d y .
$$

It is not difficult to check that

$$
\left.\partial_{t t} h(t, s, x, y)\right|_{t=0}=\frac{8 x^{2}\left(s x+\sqrt{1-s^{2}} y\right)^{2}}{\beta+x^{2}} .
$$

Thus it follows that

$$
\left.\mathbb{E} \partial_{t t} f(u)\right|_{t=0,|s| \leq 1} \gtrsim 1
$$

The desired result then follows by a simple perturbation argument using the fact that $\mathbb{E} \partial_{t t t} f$ is uniformly bounded and taking $|t|$ sufficiently small.

\section{Appendix C: technical estimates for Section 4}

Lemma C.1. Let $u=\sqrt{R} \hat{u}$ with $0<c_{1} \leq R \leq c_{2}<\infty$ and $\hat{u} \in \mathbb{S}^{n-1}$. Assume $\hat{u}=$ $\cos \theta e_{1}+\sin \theta e^{\perp}$, where $\theta \in[0, \pi]$ and $e^{\perp} \in \mathbb{S}^{n-1}$ satisfies $e^{\perp} \cdot e_{1}=0$. We have

$$
\mathbb{E} \partial_{\theta} f=a_{1}\left(\beta_{1}, \beta_{2}, R, \theta\right) \sin (2 \theta)
$$

where

$$
\gamma_{1}<a_{1}\left(\beta_{1}, \beta_{2}, R, \theta\right) \leq \gamma_{2}, \quad \forall \theta \in[0, \pi], \quad c_{1} \leq R \leq c_{2} ;
$$

and $\gamma_{1}>0, \gamma_{2}>0$ are constants depending only on $\left(\beta_{1}, \beta_{2}, c_{1}, c_{2}\right)$. Furthermore for some sufficiently small constants $\theta_{0}=\theta_{0}\left(\beta_{1}, \beta_{2}, c_{1}, c_{2}\right)>0, \theta_{1}=\theta_{1}\left(\beta_{1}, \beta_{2}, c_{1}, c_{2}\right)>0$, we have

$$
\begin{array}{ll}
\gamma_{3}<\mathbb{E} \partial_{\theta \theta} f<\gamma_{4}, & \text { if } 0 \leq \theta \leq \theta_{0} \quad \text { or } \pi-\theta_{0} \leq \theta \leq \pi, \\
\gamma_{5}<-\mathbb{E} \partial_{\theta \theta} f<\gamma_{6}, & \text { if }\left|\theta-\frac{\pi}{2}\right|<\theta_{1},
\end{array}
$$

where $\gamma_{i}>0, i=3, \cdots, 6$ depend only on $\left(\beta_{1}, \beta_{2}, c_{1}, c_{2}\right)$. 
Proof. We have

$$
\mathbb{E} f(u)=\frac{1}{2 \pi} \int_{\mathbb{R}^{2}} \frac{\left(R(x \cos \theta+y \sin \theta)^{2}-x^{2}\right)^{2}}{R+\beta_{1} R(x \cos \theta+y \sin \theta)^{2}+\beta_{2} x^{2}} e^{-\frac{x^{2}+y^{2}}{2}} d x d y .
$$

Denote

$$
h(a, b)=\frac{\left(R a^{2}-b\right)^{2}}{R+\beta_{1} R a^{2}+\beta_{2} b} .
$$

Then

$$
\begin{aligned}
& \partial_{\theta}\left(h\left(x \cos \theta+y \sin \theta, x^{2}\right)\right)=(-x \sin \theta+y \cos \theta) \partial_{a} h \\
& \partial_{x}\left(h\left(x \cos \theta+y \sin \theta, x^{2}\right)\right)=\partial_{a} h \cdot \cos \theta+2 x \partial_{b} h \\
& \partial_{y}\left(h\left(x \cos \theta+y \sin \theta, x^{2}\right)\right)=\partial_{a} h \cdot \sin \theta \\
& \partial_{\theta}\left(h\left(x \cos \theta+y \sin \theta, x^{2}\right)\right)=\left(y \partial_{x}-x \partial_{y}\right)\left(h\left(x \cos \theta+y \sin \theta, x^{2}\right)\right)-2 x y \partial_{b} h .
\end{aligned}
$$

By using integration by parts, we then obtain

$$
\begin{aligned}
\mathbb{E} \partial_{\theta} f & =\frac{1}{\pi} \int_{\mathbb{R}^{2}}(-x y)\left(\partial_{b} h\right)\left(x \cos \theta+y \sin \theta, x^{2}\right) e^{-\frac{x^{2}+y^{2}}{2}} d x d y \\
& =\frac{2}{\pi} \int_{x>0, y>0}\left(\left(\partial_{b} h\right)\left(x \cos \theta-y \sin \theta, x^{2}\right)-\left(\partial_{b} h\right)\left(x \cos \theta+y \sin \theta, x^{2}\right)\right) x y e^{-\frac{x^{2}+y^{2}}{2}} d x d y .
\end{aligned}
$$

Now denote

$$
h_{1}(a, b)=\frac{(R a-b)^{2}}{R+\beta_{1} R a+\beta_{2} b} .
$$

It is not difficult to check that for $a \geq 0, b \geq 0, \beta_{1}, \beta_{2}>0, R>0$,

$$
\partial_{a b} h_{1}=-2 R^{2} \frac{\left(1+a\left(\beta_{1}+\beta_{2}\right)\right) \cdot\left(b\left(\beta_{1}+\beta_{2}\right)+R\right)}{\left(\beta_{2} b+R+\beta_{1} a R\right)^{3}}<0 .
$$

Observe that

$$
\left(\partial_{b} h\right)(a, b)=\left(\partial_{b} h_{1}\right)\left(a^{2}, b\right) .
$$

Then if $x, y>0$ and $\theta \in[0, \pi]$, then

$$
\begin{aligned}
& \left(\partial_{b} h\right)\left(x \cos \theta-y \sin \theta, x^{2}\right)-\left(\partial_{b} h\right)\left(x \cos \theta+y \sin \theta, x^{2}\right) \\
= & \left(\partial_{b} h_{1}\right)\left((x \cos \theta-y \sin \theta)^{2}, x^{2}\right)-\left(\partial_{b} h_{1}\right)\left((x \cos \theta+y \sin \theta)^{2}, x^{2}\right) \\
= & -2 \int_{0}^{1}\left(\partial_{a b} h_{1}\right)\left((x \cos \theta+y \sin \theta)^{2}-4 \tau x y \cos \theta \sin \theta, x^{2}\right) d \tau \cdot x y \cdot \sin (2 \theta) .
\end{aligned}
$$


Integrating in $x$ and $y$, we then obtain

$$
\mathbb{E} \partial_{\theta} f=a_{1}\left(\beta_{1}, \beta_{2}, R, \theta\right) \sin (2 \theta),
$$

where $a_{1} \sim 1$ and is a smooth function of $\theta$. Differentiating in $\theta$ then gives

$$
\mathbb{E} \partial_{\theta \theta} f=2 a_{1}\left(\beta_{1}, \beta_{2}, R, \theta\right) \cos (2 \theta)+\partial_{\theta} a_{1}\left(\beta_{1}, \beta_{2}, R, \theta\right) \sin (2 \theta) .
$$

Then second term clearly vanishes near $\theta=0, \frac{\pi}{2}, \pi$. Thus the desired estimate for $\mathbb{E} \partial_{\theta \theta} f$ follows.

Lemma C.2 (Strong convexity of $\mathbb{E} f$ when $\left.\left\|u \pm e_{1}\right\| \ll 1\right)$. Let $h(u)=\mathbb{E} f(u)$. There exists $0<\epsilon_{0} \ll 1$ such that the following hold:

1. If $\left\|u-e_{1}\right\|_{2} \leq \epsilon_{0}$, then for any $\xi \in \mathbb{S}^{n-1}$, we have

$$
\sum_{i, j=1}^{n} \xi_{i} \xi_{j}\left(\partial_{i} \partial_{j} h\right)(u) \geq \gamma_{1}>0
$$

where $\gamma_{1}$ is a constant.

2. If $\left\|u+e_{1}\right\|_{2} \leq \epsilon_{0}$, then for any $\xi \in \mathbb{S}^{n-1}$, we have

$$
\sum_{i, j=1}^{n} \xi_{i} \xi_{j}\left(\partial_{i} \partial_{j} h\right)(u) \geq \gamma_{1}>0 .
$$

Proof. We shall employ the same approach as in the proof of Theorem 2.5 in the second paper of this series of work and sketch only the needed modifications. Without loss of generality consider the regime $\left\|u-e_{1}\right\|_{2} \ll 1$ and introduce the change of variables:

$$
\begin{aligned}
& u=\rho \hat{u} ; \\
& \hat{u}=\sqrt{1-s^{2}} e_{1}+s e^{\perp}, \quad e^{\perp} \cdot e_{1}=0, \quad e^{\perp} \in \mathbb{S}^{n-1},
\end{aligned}
$$

where $|\rho-1| \ll 1$ and $0 \leq s \ll 1$. Denote

$$
h_{1}(\rho, s)=h(u)=h\left(\rho\left(\sqrt{1-s^{2}} e_{1}+s e^{\perp}\right)\right),
$$

where we note that the value of $h(u)$ depends only on $(\rho, s)$. Clearly

$$
h_{1}(\rho, s)=\frac{1}{2 \pi} \int_{\mathbb{R}^{2}} \underbrace{\frac{\left(\rho^{2}\left(\sqrt{1-s^{2}} x+s y\right)^{2}-x^{2}\right)^{2}}{\rho^{2}+\beta_{1} \rho^{2}\left(\sqrt{1-s^{2}} x+s y\right)^{2}+\beta_{2} x^{2}}}_{=: h_{2}(\rho, s, x, y)} e^{-\frac{x^{2}+y^{2}}{2}} d x d y .
$$


It is easy to check that

$$
\max _{\frac{1}{2} \leq \rho \leq 2,|s| \leq \frac{1}{2}} \sum_{i, j \leq 4}\left|\partial_{\rho}^{i} \partial_{s}^{j} h_{1}(\rho, s)\right| \lesssim 1
$$

By a tedious computation, we have

$$
\partial_{\rho \rho} h_{2}(\rho, 0, x, y)=\frac{2\left(3 \rho^{2}+\rho^{6}\right) x^{4}+k_{1} \cdot x^{6}+k_{2} x^{8}}{\left(\beta_{2} x^{2}+\rho^{2}\left(1+\beta_{1} x^{2}\right)\right)^{3}}
$$

where

$$
\begin{aligned}
& k_{1}=2\left(-\beta_{2}+6 \beta_{1} \rho^{2}+6 \beta_{2} \rho^{2}+3 \beta_{2} \rho^{4}+2 \beta_{1} \rho^{6}\right) \\
& k_{2}=2\left(-\beta_{1} \beta_{2}-2 \beta_{2}^{2}+3 \beta_{1}^{2} \rho^{2}+6 \beta_{1} \beta_{2} \rho^{2}+6 \beta_{2}^{2} \rho^{2}+3 \beta_{1} \beta_{2} \rho^{4}+\beta_{1}^{2} \rho^{6}\right) .
\end{aligned}
$$

Since $\rho \rightarrow 1$, it is clear that $k_{1}>0$ and $k_{2}>0$, and thus

$$
\partial_{\rho \rho} h_{1}(1,0) \gtrsim 1
$$

It is not difficult to check that $\partial_{s} h_{1}(\rho, 0)=0$ for any $\rho>0$. Clearly also $\partial_{\rho s} h_{1}(\rho, 0)=0$ for any $\rho>0$. To compute $\partial_{s s} h_{1}(1,0)$ we shall use Lemma C.1. Observe that $(s=\sin \theta$ with $\theta \rightarrow 0+$ )

$$
\begin{aligned}
& h_{1}(\rho, \sin \theta)=\mathbb{E} f(u) \\
& \cos \theta \partial_{s} h_{1}(\rho, \sin \theta)=\mathbb{E} \partial_{\theta} f ; \\
& -\sin \theta \partial_{s} h_{1}(\rho, \sin \theta)+\cos ^{2} \theta \partial_{s s} h_{1}(\rho, \sin \theta)=\mathbb{E} \partial_{\theta \theta} f .
\end{aligned}
$$

Clearly it follows that

$$
\partial_{s s} h_{1}(1,0) \gtrsim 1 \text {. }
$$

The rest of the argument is then essentially the same as in the proof of Theorem 2.5 in the second paper. We omit further details.

Proof of Theorem 4.3. We rewrite

$$
f(u)=\frac{1}{m} \sum_{k=1}^{m} G\left(\|u\|_{2}^{2},\left(a_{k} \cdot u\right)^{2}, X_{k}^{2}\right),
$$

where

$$
G(a, b, c)=\frac{(b-c)^{2}}{a+\beta_{1} b+\beta_{2} c} .
$$


Clearly for any $\xi \in \mathbb{S}^{n-1}$,

$$
\begin{aligned}
& \sum_{i, j=1}^{n} \xi_{i} \xi_{j} \partial_{u_{i} u_{j}} f \\
= & \frac{1}{m} \sum_{k=1}^{m} \partial_{a} G \cdot 2\|\xi\|_{2}^{2} \\
& +\frac{1}{m} \sum_{k=1}^{m} \partial_{a a} G \cdot 4(u \cdot \xi)^{2} \\
& +\frac{1}{m} \sum_{k=1}^{m} \partial_{a b} G \cdot 8\left(a_{k} \cdot u\right)\left(a_{k} \cdot \xi\right)(\xi \cdot u) \\
& +\frac{1}{m} \sum_{k=1}^{m} \partial_{b b} G \cdot 4\left(a_{k} \cdot u\right)^{2}\left(a_{k} \cdot \xi\right)^{2} \\
& +\frac{1}{m} \sum_{k=1}^{m} \partial_{b} G \cdot 2\left(a_{k} \cdot \xi\right)^{2} .
\end{aligned}
$$

In the above,

$$
\partial_{a} G=\left(\partial_{a} G\right)\left(\|u\|_{2}^{2},\left(a_{k} \cdot u\right)^{2}, X_{k}^{2}\right)
$$

and similar notation is used for $\partial_{a a} G, \partial_{b b} G, \partial_{b} G$.

Estimate of (C.1) and (C.2). Clearly these two terms are OK for union bounds, and we have (for $m \gtrsim n$ and with high probability)

$$
\mid(\text { C.1 })-\text { mean }|+|(\text { C.2 })-\text { mean } \mid \ll 1, \quad \forall \xi \in \mathbb{S}^{n-1}, \quad \forall \frac{1}{2} \leq\|u\|_{2} \leq 2 .
$$

Estimate of (C.3). We have

$$
\left(\partial_{a b} G\right)(a, b, c)=-\frac{2(b-c)\left(a+\left(\beta_{1}+\beta_{2}\right) c\right)}{\left(a+\beta_{1} b+\beta_{2} c\right)^{3}} .
$$

Consider the function

$$
\tilde{G}_{1}(a, y, c)=-y \frac{2\left(y^{2}-c\right)\left(a+\left(\beta_{1}+\beta_{2}\right) c\right)}{\left(a+\beta_{1} y^{2}+\beta_{2} c\right)^{3}} .
$$

Clearly for $\frac{1}{10} \leq a, \tilde{a} \leq 10, y, \tilde{y} \in \mathbb{R}, c \geq 0$, we have $\left|\tilde{G}_{1}\right| \lesssim 1$ and

$$
\left|\tilde{G}_{1}(a, y, c)-\tilde{G}_{1}(\tilde{a}, \tilde{y}, c)\right| \lesssim|a-\tilde{a}|+|y-\tilde{y}| .
$$


Then for any $(u, \tilde{u})$ with $\frac{1}{2} \leq\|u\|_{2},\|\tilde{u}\|_{2} \leq 2$ and $(\xi, \tilde{\xi})$ with $\xi, \tilde{\xi} \in \mathbb{S}^{n-1}$, we have

$$
\begin{aligned}
& \mid\left(\partial_{a b} G\right)\left(\|u\|_{2}^{2},\left(a_{k} \cdot u\right)^{2}, X_{k}^{2}\right)\left(a_{k} \cdot u\right)\left(a_{k} \cdot \xi\right) \\
& -\left(\partial_{a b} G\right)\left(\|\tilde{u}\|_{2}^{2},\left(a_{k} \cdot \tilde{u}\right)^{2}, X_{k}^{2}\right)\left(a_{k} \cdot \tilde{u}\right)\left(a_{k} \cdot \tilde{\xi}\right) \mid \\
\lesssim & \left|a_{k} \cdot(\xi-\tilde{\xi})\right|+\left|a_{k} \cdot \xi\right| \cdot\left(\left|a_{k} \cdot(u-\tilde{u})\right|+\|u-\tilde{u}\|_{2}\right) .
\end{aligned}
$$

Thus the union bound is also OK for this term, and we have

$$
\mid(\mathrm{C} .3)-\text { mean } \mid \ll 1, \quad \forall \xi \in \mathbb{S}^{n-1}, \quad \forall \frac{1}{2} \leq\|u\|_{2} \leq 2 .
$$

Estimate of (C.4) and (C.5). We begin by noting that (C.4) and (C.5) can be combined into one term. Namely, observe that

$$
\left(\partial_{b b} G\right)(a, b, c) \cdot 2 b+\left(\partial_{b} G\right)(a, b, c)=\frac{H_{1}}{\left(a+\beta_{1} b+\beta_{2} c\right)^{3}},
$$

where

$$
\begin{gathered}
H_{1}=\beta_{1}^{2} b^{3}+a^{2}(6 b-2 c)+3 \beta_{1} \beta_{2} b^{2} c+3 b\left(\beta_{1}^{2}+2 \beta_{1} \beta_{2}+2 \beta_{2}^{2}\right) c^{2}-\beta_{2}\left(\beta_{1}+2 \beta_{2}\right) c^{3} \\
+a\left(3 \beta_{1} b^{2}+6\left(\beta_{1}+2 \beta_{2}\right) b c-\left(\beta_{1}+4 \beta_{2}\right) c^{2}\right) .
\end{gathered}
$$

We can then write

$$
(\mathrm{C} .4)+(\mathrm{C} .5)=\frac{1}{m} \sum_{k=1}^{m}\left(a_{k} \cdot \xi\right)^{2} h_{3}\left(u, a_{k} \cdot u, X_{k}\right),
$$

where $h_{3}$ is a bounded smooth function with bounded derivatives in all of its arguments. Now let $\phi \in C_{c}^{\infty}$ be such that $0 \leq \phi(x) \leq 1$ for all $x, \phi(x)=1$ for $|x| \leq 1$ and $\phi(x)=0$ for $|x| \geq 2$. We then split the sum as

$$
\begin{aligned}
& \frac{1}{m} \sum_{k=1}^{m}\left(a_{k} \cdot \xi\right)^{2} h_{3}\left(u, a_{k} \cdot u, X_{k}\right), \\
= & \frac{1}{m} \sum_{k=1}^{m}\left(a_{k} \cdot \xi\right)^{2} \phi\left(\frac{a_{k} \cdot \xi}{K}\right) h_{3}\left(u, a_{k} \cdot u, X_{k}\right) \\
& +\frac{1}{m} \sum_{k=1}^{m}\left(a_{k} \cdot \xi\right)^{2}\left(1-\phi\left(\frac{a_{k} \cdot \xi}{K}\right)\right) \cdot h_{3}\left(u, a_{k} \cdot u, X_{k}\right),
\end{aligned}
$$


where $K$ will be taken sufficiently large. Clearly the first term will be OK for union bounds. On the other hand, the second term can be dominated by

$$
\text { const } \cdot \frac{1}{m} \sum_{k=1}^{m}\left(a_{k} \cdot \xi\right)^{2}\left(1-\phi\left(\frac{a_{k} \cdot \xi}{K}\right)\right)
$$

which can be made small by taking $K$ large. Thus we have

$$
|(\mathrm{C} .4)+(\mathrm{C} .5)-\operatorname{mean}| \ll 1, \quad \forall \xi \in \mathbb{S}^{n-1}, \quad \forall \frac{1}{2} \leq\|u\|_{2} \leq 2 .
$$

Collecting the estimates, we have for $m \gtrsim n$ and with high probability,

$$
\mid \sum_{i, j=1}^{n} \xi_{i} \xi_{j} \partial_{u_{i} u_{j}} f(u)-\text { mean } \mid \ll 1, \quad \forall \xi \in \mathbb{S}^{n-1}, \quad \forall \frac{1}{2} \leq\|u\|_{2} \leq 2 .
$$

The desired result then follows from Lemma C.2.

\section{Acknowledgements}

J. F. Cai was supported in part by Hong Kong Research Grant Council General Research Grant Nos. 16309518, 16309219, 16310620, and 16306821. Y. Wang was supported in part by Hong Kong Research Grant Council General Research Grant Nos. 16306415 and 16308518.

\section{References}

[1] S. Bhojanapalli, N. Behnam, and N. Srebro, Global optimality of local search for low rank matrix recovery, Adv. Neural Infor. Proc. Syst., (2016), pp. 3873-3881.

[2] E. J. Candès and X. Li, Solving quadratic equations via PhaseLift when there are about as many equations as unknowns, Found. Comput. Math., 14(5) (2014), pp. $1017-1026$.

[3] E. J. Candès, X. Li, and M. Soltanolkotabi, Phase retrieval via Wirtinger flow: Theory and algorithms, IEEE Trans. Inf. Theory, 61(4) (2015), pp. 1985-2007.

[4] E. J. Candès, T. Strohmer, and V. Voroninski, Phaselift: Exact and stable signal recovery from magnitude measurements via convex programming, Commun. Pure Appl. Math., 66(8) (2013), pp. 1241-1274.

[5] J. Cai, M. Huang, D. Li and Y. Wang, Solving phase retrieval with random initial guess is nearly as good as by spectral initialization, Appl. Comput. Harmon. Anal., (2021). 
[6] J. Cai, M. Huang, D. Li and Y. Wang, Nearly optimal bounds for the global geometric landscape of phase retrieval, arxiv preprint, in preparation, 2021.

[7] Y. Chen and E. J. Candès, Solving random quadratic systems of equations is nearly as easy as solving linear systems, Commun. Pure Appl. Math., 70(5) (2017), pp. $822-883$.

[8] J. C. Dainty and J. R. Fienup, Phase retrieval and image reconstruction for astronomy, Image Recovery: Theory and Application, 231 (1987), 275.

[9] S. S. Du, C. Jin, J. D. Lee, and M. I. Jordan, Gradient descent can take exponential time to escape saddle points, Adv. Neural Infor. Proc. Syst., (2017), pp. 1067-1077.

[10] J. R. Fienup, Phase retrieval algorithms: a comparison, Appl. Opt., 21(15) (1982), pp. 2758-2769.

[11] B. Gao, Y. Wang, and Z. Xu, Solving a perturbed amplitude-based model for phase retrieval, 2019, available: http://arxiv.org/abs/1904.10307.

[12] B. Gao and Z. Xu, Phaseless recovery using the Gauss-Newton method, IEEE Trans. Signal Process., 65(22) (2017), pp. 5885-5896.

[13] R. Ge, F. Huang, C. Jin, and Y. Yuan, Escaping from saddle points - online stochastic gradient for tensor decomposition, Conference on Learning Theory, (2015), pp. 797842.

[14] R. Ge, J. Lee, C. Jin, and T. Ma, Matrix completion has no spurious local minimum, Adv. Neural Infor. Proc. Syst., (2016), pp. 2973-2981.

[15] R. W. Gerchberg, A practical algorithm for the determination of phase from image and diffraction plane pictures, Optik, 35 (1972), pp. 237-246.

[16] R. W. Gerchberg and W. O. Saxton, A practical algorithm for the determination of the phase from image and diffraction plane pictures, Optik, 35 (1972), pp. 237-246.

[17] R. W. Harrison, Phase problem in crystallography, Josa A, 10(5) (1993), pp. 10461055.

[18] M. Huang and Y. Wang, Linear convergence of randomized Kaczmarz method for solving complex-valued phaseless equations, 2021, available: http://arxiv.org/abs/2109.11811.

[19] C. Jin, R. Ge, P. Netrapalli, S. M. Kakade, and M. I. Jordan, How to escape saddle points efficiently, Proceedings of the 34th International Conference on Machine Learning-Volume 70, (2017), pp. 1724-1732.

[20] C. Jin, P. Netrapalli, and M. I. Jordan, Accelerated gradient descent escapes saddle points faster than gradient descent, 2017, available: http://arxiv.org/abs/1711.10456.

[21] Z. Li, J. F. Cai, and K. Wei, Towards the optimal construction of a loss function without spurious local minima for solving quadratic equations, IEEE Trans. Inf. Theory, 66(5) (2020), pp. 3242-3260.

[22] J. Miao, T. Ishikawa, Q. Shen, and T. Earnest, Extending x-ray crystallography to allow the imaging of noncrystalline materials, cells, and single protein complexes, Annu. Rev. Phys. Chem., (59) (2008), pp. 387-410.

[23] R. P. Millane, Phase retrieval in crystallography and optics, J. Optical Soc. Am. A, 
7(3) (1990), pp. 394-411.

[24] P. Netrapalli, P. Jain, and S. Sanghavi, Phase retrieval using alternating minimization, IEEE Trans. Signal Process., 63(18) (2015), pp. 4814-4826.

[25] D. Park, A. Kyrillidis, and C. Caramanis, Non-square matrix sensing without spurious local minima via the Burer-Monteiro approach, 2016, available: http://arxiv.org/abs/1609.03240.

[26] H. Sahinoglou and S. D. Cabrera, On phase retrieval of finite-length sequences using the initial time sample, IEEE Trans. Circuits and Syst., 38(8) (1991), pp. 954-958.

[27] Y. Shechtman, Y. C. Eldar, O. Cohen, H. N. Chapman, J. Miao, and M. Segev, Phase retrieval with application to optical imaging: a contemporary overview, IEEE Signal Process. Mag., 32(3) (2015), pp. 87-109.

[28] J. Sun, Q. Qu, and J, Wright, A geometric analysis of phase retrieval, Found. Comput. Math., 18(5) (2018), pp. 1131-1198.

[29] J. Sun, Q. Qu, and J, Wright, Complete dictionary recovery over the sphere I: Overview and the geometric picture, IEEE Trans. Inf. Theory, 63(2) (2016), pp. 853-884.

[30] Y. S. Tan and R. Vershynin, Phase retrieval via randomized kaczmarz: Theoretical guarantees, Information and Inference: A Journal of the IMA, 8(1) (2019), pp. 97123.

[31] R. Vershynin, High-Dimensional Probability: An Introduction with Applications in Data Science, U.K. Cambridge University Press, 2018.

[32] I. Waldspurger, A. d'Aspremont, and S. Mallat, Phase recovery, maxcut and complex semidefinite programming, Math. Prog., 149(1-2) (2015), pp. 47-81.

[33] A. Walther, The question of phase retrieval in optics, J. Mod. Opt., 10(1) (1963), pp. 41-49.

[34] G. Wang, G. B. Giannakis, and Y. C. Eldar, Solving systems of random quadratic equations via truncated amplitude flow, IEEE Trans. Inf. Theory, 64(2) (2018), pp. 773-794.

[35] K. Wei, Solving systems of phaseless equations via kaczmarz methods: a proof of concept study, Inverse Probl., 31(12) (2015), 125008.

[36] H. Zhang, Y. Zhou, Y. Liang, and Y. Chi, A nonconvex approach for phase retrieval: Reshaped wirtinger flow and incremental algorithms, The Journal of Machine Learning Research, 18(1) (2017), pp. 5164-5198. 